\title{
Solution of the Dynamic Interaction Problem between a Framed Structure and an Acoustic Cavity Using Imposed Deformation Functions at the Interface
}

\author{
Paulo Marcelo Vieira Ribeiro and Lineu José Pedroso \\ Department of Civil Engineering, University of Brasília, 70919-970 Brasília, DF, Brazil \\ Correspondence should be addressed to Paulo Marcelo Vieira Ribeiro, pauloribeiro@unb.br
}

Received 14 August 2009; Revised 17 February 2010; Accepted 23 March 2010

Academic Editor: Horst Ecker

Copyright (C) 2010 P. M. V. Ribeiro and L. J. Pedroso. This is an open access article distributed under the Creative Commons Attribution License, which permits unrestricted use, distribution, and reproduction in any medium, provided the original work is properly cited.

This article presents an analytical procedure for solution of the dynamic interaction problem of a vibrating framed structure connected to a bidimensional cavity, containing an acoustic fluid. Initially the pressure solution for the fluid domain is developed, using the separation of variables technique. In a next step, this solution is applied to an entirely open cavity and to a closed cavity in the transversal direction, both containing a vibrating boundary with an arbitrary deformation. The generalized parameters of the structure (mass, rigidity, and force) are obtained by means of the virtual work principle, with the generalized force represented by the dynamic pressures acting on the interface. The dynamic equilibrium equation of the system is established for an imposed deformation, making a parametric study of the involved variables possible. Finally, it is demonstrated that this procedure can be generalized, allowing the construction of practical abacuses for other boundary conditions of both the structure and the cavity, and that these results allow a reasonable interpretation of the coupling regions, including the prediction of added mass and added stiffness effects, as well as corresponding frequencies and mode shapes of the coupled problem.

\section{Introduction}

The problems of dynamic fluid-structure interactions are of great interest to a wide range of research fields in engineering. In these cases, the movement of these two subsystems is not independent and is governed by dynamic contact conditions. According to Bathe [1], among the categories of coupled problems, the interaction between a structure and an acoustic fluid is distinguished for its simplicity. The inevitable contact of the structures with the acoustic medium (air, water, etc.) makes the acoustic-structural interaction relevant in the analysis of many physical systems [2]. 
The problems of fluid contained in enclosures are common in many practical applications. Great interest has been devoted to the acoustic comfort of passengers in aircraft or automobile cabins, for example. Additionally the case of water cooled nuclear reactors, rocket propeller tanks, submerged sonar domes, and reservoirs can also be cited as relevant examples of this category of problem [3].

Fluid-structure interaction problems involving acoustic cavities have already been solved by various methods, including analytical, semianalytical, and numerical approaches [4-9]. In the majority of these works the acoustic medium was represented by the air. Among the studies involving fluid contained in tanks or reservoirs, one can cite the studies of [10-13], which evaluated the interaction effects between the fluid and a vibrating column, and [1416], which established the coupling effects between a plate and the surrounding fluid. In some of these works the problem was solved analytically, with the determination of frequencies and mode shapes of the coupled problem. However, little attention has been given to the development of abacus and graphical representations that allows the interpretation of such phenomena, including the identification of added mass and added stiffness regions, as well as physical interpretation of the corresponding mode shapes and its relation with the cavity modes.

One of the greatest advantages of the analytical treatment of coupled problems is the analysis of dimensionless parameters, which allow physical interpretation of the solutions. However, for more complex models, such set of solutions is often not possible. These are applied only to some specific cases, while numerical procedures can handle mode general problems. However, the implementation of numerical solutions is time consuming both in construction and in computer processing. According to Amabili and Kwak [15], analytical procedures show its importance in the solution of simple cases and can be used in the validation of numerical solutions.

In this paper an analytical procedure applied to a framed structure coupled to a bidimensional acoustic cavity is developed for solution of frequencies and mode shapes of the coupled problem. The separation of variables technique is employed, resulting in a general pressure solution, which is then applied to specific cases of an entirely open cavity and a closed cavity in the transversal direction, both containing a vibrating boundary. The association of this movement with the related structural vibration function, and the introduction of the dynamic fluid pressures as external forces, enables the construction of the dynamic equilibrium equation of the coupled structure, which is solved for frequencies of the equivalent system. While this procedure presents the limitation of prior knowledge of the boundary deformation, the mathematical simplicity of the solution justifies its application, resulting in equations represented with clear and well-defined parameters that allow the construction of abacuses for interpretation of frequencies and mode shapes.

\section{Fluid Domain General Solution}

The basic assumptions of this problem are the corresponding to treatment of this medium as an acoustic fluid. With these considerations, it is assumed that the fluid transmits only pressure waves. Some applications of this theory include propagation of pressure waves in pipes and sound waves propagating through fluid-solid media. The following basic assumptions are made for the solution of this problem (based on the propositions of Chopra [17] and Rashed [18]). 
(i) The fluid is homogeneous, inviscid, and linearly compressible.

(ii) The flow is irrotational.

(iii) Displacements and their derivatives are small.

(iv) Surface wave effects are neglected.

(v) The movement of the fluid-structure interface is bidimensional (the same for any vertical plane perpendicular to the structural axis).

(vi) The fluid-structure interface is vertical.

(vii) Interface displacements are represented by an arbitrary deformation function.

The previous assumptions lead to a dynamic pressure distribution $p(x, y, t)$, in excess of the static pressure, given by

$$
\nabla^{2} p=\frac{1}{c^{2}} \frac{\partial^{2} p}{\partial t^{2}}
$$

which corresponds to the bidimensional wave equation, where

$$
\nabla^{2}=\frac{\partial^{2}}{\partial x^{2}}+\frac{\partial^{2}}{\partial y^{2}}
$$

is the bidimensional Laplacian operator and $c=\sqrt{\mathcal{K} / \rho_{f}}$ represents the fluid sound velocity, where $\mathcal{K}$ indicates the fluid bulk modulus and $\rho_{f}$ its density. For an incompressible fluid $\mathcal{K} \rightarrow \infty$, and therefore $c \rightarrow \infty$, thus (2.1) is reduced to

$$
\nabla^{2} p=0
$$

This indicates Laplace's equation governing the dynamic pressures in an incompressible fluid. It is evident that this expression is a particular case of (2.1).

Solution of (2.1) is achieved using the separation of variables technique. Therefore it is assumed that this expression can be separated, resulting in

$$
p(x, y, t)=F(x) G(y) T(t) .
$$

Substitution of (2.4) in (2.1) provides

$$
F^{\prime \prime}(x) G(y) T(t)+F(x) G^{\prime \prime}(y) T(t)=\frac{1}{c^{2}} F(x) G(y) T^{\prime \prime}(t),
$$

where the tiles indicate derivatives related to the corresponding variable. Division of (2.5) by $F(x) G(y) T(t)$ results in

$$
-\frac{G^{\prime \prime}(y)}{G(y)}=\frac{F^{\prime \prime}(x)}{F(x)}-\frac{1}{c^{2}} \frac{T^{\prime \prime}(t)}{T(t)}
$$


Analysis of expression (2.6) indicates that the left-hand side of this equation depends only on $y$, while the right-hand side depends on $x$ and $t$. Since this equation must be satisfied for any values of $x, y$, and $t$, it is necessary that both sides are equivalent to an arbitrary constant $\beta$, which can assume positive, negative, or null values. Thus,

$$
-\frac{G^{\prime \prime}(y)}{G(y)}=\frac{F^{\prime \prime}(x)}{F(x)}-\frac{1}{c^{2}} \frac{T^{\prime \prime}(t)}{T(t)}=\beta
$$

This last expression provides three ordinary differential equations:

$$
\begin{aligned}
& -\frac{G^{\prime \prime}(y)}{G(y)}=\beta, \\
& \frac{F^{\prime \prime}(x)}{F(x)}=\beta+\frac{1}{c^{2}} \frac{T^{\prime \prime}(t)}{T(t)}=\alpha, \\
& \frac{T^{\prime \prime}(t)}{T(t)}=c^{2}\left[\frac{F^{\prime \prime}(x)}{F(x)}-\beta\right]=\delta,
\end{aligned}
$$

where $\alpha$ and $\delta$ represent arbitrary separation constants. Equations (2.9) and (2.10) provide the following relation:

$$
\alpha=\beta+\frac{1}{c^{2}} \delta
$$

The combined solutions of the differential equations (2.8) to (2.10) provide the complete solution of (2.1). However, it is important to notice that each one of these expressions requires two constants, resulting in a total of six unknown constants. A simple and time independent solution for this problem can be achieved with the hypothesis of time harmonic vibrations, with frequency $\omega$. Thus it is assumed that the time-related function is given by

$$
T(t)=e^{-i \omega t}
$$

The hypothesis of time harmonic travelling waves establishes an important relation between the separation constants in the $x$ and $y$ directions. Substitution of (2.12) in (2.10) provides

$$
\frac{T^{\prime \prime}(t)}{T(t)}=-\omega^{2}=\delta
$$

Substitution of (2.13) in (2.7) gives

$$
-\frac{G^{\prime \prime}(y)}{G(y)}=\frac{F^{\prime \prime}(x)}{F(x)}+\frac{\omega^{2}}{c^{2}}=\beta .
$$


Table 1: Resume of possible solutions according to $\beta$.

\begin{tabular}{lccc}
\hline & $\alpha$ & $G(y)$ & $F(x)$ \\
\hline$\beta=0$ & $\frac{\omega}{c}$ & $C_{1}+C_{2} y$ & $C_{3} \cdot e^{i \alpha x}+C_{4} \cdot e^{-i \alpha x}$ \\
or $^{*}$
\end{tabular}

${ }^{*}$ Valid only for nonnull constants forming a complex conjugated pair.

Thus

$$
\frac{F^{\prime \prime}(x)}{F(x)}=\beta-\frac{\omega^{2}}{c^{2}}
$$

From this last expression it can be concluded that

$$
\alpha=\beta-\frac{\omega^{2}}{c^{2}} .
$$

Equation (2.16) indicates that the hypothesis of time harmonic travelling waves results in a separation constant in the $x$ direction that depends on the following parameters: frequency $(\omega)$, fluid sound velocity $(c)$, and $y$ direction separation constant $(\beta)$.

The solution of (2.1) can be established with the solution of the two ordinary differential equations given by (2.14) and (2.15). The separation constant $\beta$ can assume positive, negative, or null values. However, it should be noticed that depending on the problem, some of these solutions may result in expressions that are either trivial or without physical meaning.

Table 1 presents a resume of the possible solutions in both $x$ and $y$ directions according to the value of $\beta$. Values of $\alpha$ are also included.

\section{Solution of Rectangular Cavities with a Vibrating Boundary}

\subsection{Entirely Open Cavity}

In this case it is assumed that the cavity has the following pair of boundary conditions in the $x$ and $y$ directions, respectively: vibrating boundary-open and open-open. The vibrating boundary condition is related to the fluid-structure interaction at the interface, while the open condition implies at zero pressure at the contour. It will be shown later that this cavity, despite the lack of physical meaning, presents mathematical simplications that justify the use of such conditions, providing a useful interpretation of the phenomena. Figure 1 illustrates the analyzed domain as well as the boundary conditions. 


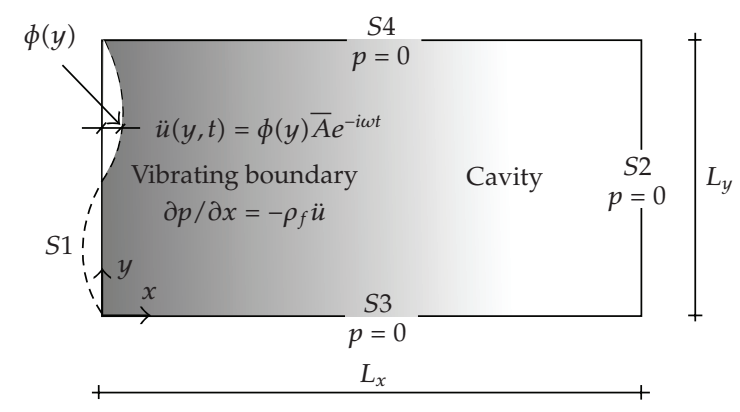

Figure 1: Analyzed domain scheme including boundary conditions.

It is assumed that the vibrating boundary horizontal acceleration is governed by a time harmonic function, related to an arbitrary shape function $\phi(y)$ and a maximum amplitude $\bar{A}$. Thus, for a given point at the interface the corresponding horizontal acceleration will be given by

$$
\ddot{u}(y, t)=\phi(y) \bar{A} e^{-i \omega t} .
$$

Equations (2.16) and (3.1) can be substituted at the interface boundary condition $S 1$. Thus

$$
\left.S 1 \rightarrow \frac{\partial p}{\partial x}\right|_{x=0}=-\rho_{f} \ddot{u}
$$

Therefore

$$
\left.S 1 \longrightarrow \frac{\partial P}{\partial x}\right|_{x=0}=-\rho_{f} \phi(y) \bar{A}
$$

The remaining boundary conditions are easily established, indicating a zero pressure at the contour. Therefore, in order to avoid the trivial solution, $P(x, y)=F(x) G(y)$ must be null at these locations. Thus

$$
\begin{aligned}
& S 2 \longrightarrow p\left(L_{x}, y, t\right)=0 \therefore F\left(L_{x}\right) G(y)=0 \therefore F\left(L_{x}\right)=0, \\
& S 3 \longrightarrow p(x, 0, t)=0 \therefore F(x) G(0)=0 \therefore G(0)=0, \\
& S 4 \longrightarrow p\left(x, L_{y}, t\right)=0 \therefore F(x) G\left(L_{y}\right)=0 \therefore G\left(L_{y}\right)=0 .
\end{aligned}
$$

Equations (3.3) and (3.4) define the four boundary conditions needed for the solution of this problem. Table 1 will be used as guide for selection of the corresponding longitudinal $F(x)$ and transversal $G(y)$ solutions. Nontrivial solutions occur only when $\beta=\kappa^{2}$. For this value the transversal solution $G(y)$ is given by

$$
G(y)=B \cdot \sin (\kappa \cdot y),
$$


where,

$$
\kappa=\frac{n \pi}{L_{y}}, \quad n=1,2,3 \ldots
$$

The corresponding longitudinal solution for this value of $\beta$ is given by

$$
F(x)=D \cdot\left[\sin (\sqrt{\alpha} \cdot x)-\tan \left(\sqrt{\alpha} \cdot L_{x}\right) \cdot \cos (\sqrt{\alpha} \cdot x)\right],
$$

where the separation constant $\alpha$ is defined by

$$
\alpha_{n}=\left(\frac{\omega}{c}\right)^{2}-\left(\frac{n \pi}{L_{y}}\right)^{2}, \quad n=1,2,3 \ldots
$$

The complete solution is given by the sum of every possible solution of $P_{n}(x, y)$. Thus

$$
P(x, y)=\sum_{n=1}^{\infty} E_{n} \cdot\left[\sin \left(\sqrt{\alpha_{n}} \cdot x\right)-\tan \left(\sqrt{\alpha_{n}} \cdot L_{x}\right) \cdot \cos \left(\sqrt{\alpha_{n}} \cdot x\right)\right] \sin \left(\kappa_{n} \cdot y\right) .
$$

The remaining constant $E_{n}$ is obtained with application of boundary condition (3.3), using the sine function orthogonality property. Thus

$$
E_{n}=-\frac{2 \rho_{f} \bar{A}}{L_{y} \sqrt{\alpha_{n}}} \int_{0}^{L_{y}} \phi(y) \sin \left(\kappa_{n} \cdot y\right) d y .
$$

Equation (3.10) can be substituted in (3.9) resulting in

$$
\begin{aligned}
P(x, y)= & -\frac{2 \rho_{f} \bar{A}}{L_{y}} \sum_{n=1}^{\infty} \frac{1}{\sqrt{\alpha_{n}}} \int_{0}^{L_{y}} \phi(y) \sin \left(\kappa_{n} \cdot y\right) d y \\
& \cdot\left[\sin \left(\sqrt{\alpha_{n}} \cdot x\right)-\tan \left(\sqrt{\alpha_{n}} \cdot L_{x}\right) \cdot \cos \left(\sqrt{\alpha_{n}} \cdot x\right)\right] \sin \left(\kappa_{n} \cdot y\right) .
\end{aligned}
$$

This last expression represents the dynamic pressure field solution for an open bidimensional cavity containing a vibrating boundary subjected to a harmonic motion, described by an arbitrary shape function $\phi(y)$.

\section{Application of Sinusoidal Deformation Functions on S1 Boundary}

The sine function orthogonality property can be applied once again, if it is assumed that the vibrating boundary $S 1$ has the following shape function:

$$
\phi(y)=\sin \left(\frac{j \pi y}{L_{y}}\right), \quad j=1,2,3, \ldots,
$$


which could be associated (in the case where the vibrating boundary represents deformations of a framed structure) to the corresponding mode shapes of a simple beam. In this case, (3.11) will result in

$$
P(x, y)=-\frac{\rho_{f} \bar{A}}{\sqrt{\alpha_{j}}} \cdot \frac{\sin \left[\sqrt{\alpha_{j}}\left(x-L_{x}\right)\right]}{\cos \left(\sqrt{\alpha_{j}} \cdot L_{x}\right)} \cdot \sin \left(\frac{j \pi y}{L_{y}}\right) .
$$

Defining $\omega=\chi \pi c / L_{y}$, where $\chi$ is an arbitrary value, and substituting this expression in (3.8)

$$
\alpha_{j}=\left(\frac{x \pi}{L_{y}}\right)^{2}-\left(\frac{j \pi}{L_{y}}\right)^{2}=\left(\frac{\pi}{L_{y}}\right)^{2} \cdot\left(x^{2}-j^{2}\right) .
$$

Therefore, applying (3.14) in (3.13) gives

$$
P\left(\xi_{x}, \xi_{y}\right)=-\frac{\rho_{f} \bar{A} L_{y}}{\pi} \cdot \frac{\sin \left[\pi \sqrt{x^{2}-j^{2}} \cdot\left(\xi_{x}-1\right) \cdot r\right]}{\sqrt{x^{2}-j^{2}} \cdot \cos \left(\pi \sqrt{x^{2}-j^{2}} \cdot r\right)} \cdot \sin \left(j \pi \xi_{y}\right)
$$

where

$$
\xi_{x}=\frac{x}{L_{x}}, \quad \xi_{y}=\frac{y}{L_{y}}, \quad r=\frac{L_{x}}{L_{y}}
$$

This last expression can be rewritten in terms of a longitudinal function $f_{1}$, a transversal function $s_{1}$, and an amplitude factor $q$. Thus

$$
P=-\frac{\rho_{f} \bar{A} L_{y}}{\pi} \cdot \frac{f_{1}\left(j, x, \xi_{x}, r\right)}{q(j, x, r)} \cdot s_{1}\left(j, \xi_{y}\right)
$$

It is important to notice the presence of a cosine function in $q$. This term will lead expression (3.17) to infinite results whenever the value of this trigonometric function approaches zero. This condition establishes critical points that result in resonance responses of the acoustic cavity. These points are defined by

$$
X_{\text {critical }}=\sqrt{\left(\frac{m}{2 r}\right)^{2}+j^{2}}, \quad m=1,3,5 \ldots
$$

This last expression indicates that every value of $j$ is associated with infinite critical values that will lead the pressure response to infinity. These values will always occur for $x>j$. The limit of $f_{1} s_{1} / q$ when $x \rightarrow X_{\text {critical }}$ is given by

$$
\lim _{x \rightarrow x_{\text {critical }}} \frac{f_{1} s_{1}}{q}=\left[-\frac{2 r}{m} \cdot \tan \left(\frac{m \pi}{2}\right)\right] \cdot \cos \left(\frac{m \pi \xi_{x}}{2}\right) \cdot \sin \left(j \pi \xi_{y}\right) .
$$


Equations (3.18) and (3.19) are equivalent, respectively, to the frequencies and mode shapes of an acoustic cavity, closed-opened in the longitudinal direction $x$, and opened-opened in the transversal direction $y$. Therefore, it can be concluded that critical values represent a set of frequencies that will lead to the mode shapes of the associated cavity, with the vibrating boundary condition at $S 1$ replaced by a rigid wall. Table 2 indicates the first seven uncoupled modes for cavities with $r=1 / 2,1$, and 2 . The corresponding mode shapes of the vibrating boundary for a given value of $j$ are also indicated.

Analysis of Table 2 results indicates that a given mode with an arbitrary value of $j$ will produce only $j$ circular zones in the transversal direction. For example, solutions with two circular regions in $y$ are always associated to $j=2$. In the longitudinal direction the corresponding relation is given by $m / 2$. Thus, for $m=3$, for example, only one and a half circular regions are expected at the $x$ direction. Cavities with a greater value of $r$ will present mode sequences with lower values of $j$, while smaller values of $r$ will provide sequences with higher values of this parameter.

\subsection{Closed Cavity in the Transversal Direction}

In this case boundary conditions at $S 3$ and $S 4$ are replaced by

$$
\left.S 3 \longrightarrow \frac{\partial p}{\partial x}\right|_{y=0}=0,\left.\quad S 4 \longrightarrow \frac{\partial p}{\partial x}\right|_{y=L_{y}}=0
$$

Nontrivial solutions are defined by the following values of $G(y)$ :

$$
G^{\prime}(0)=0, \quad G^{\prime}\left(L_{y}\right)=0 .
$$

For $\beta=0$ boundary conditions given by (3.21) provide a valid solution, given by Table 1 . Thus

$$
G(y)=C_{1}
$$

Table 1 provides the correspondent longitudinal solution, defined by

$$
F(x)=B\left[\sin (\alpha x)-\tan \left(\alpha L_{x}\right) \cos (\alpha x)\right]
$$

Therefore, the complete solution for $\beta=0$ is given by

$$
P(x)=\bar{C}\left[\sin (\alpha x)-\tan \left(\alpha L_{x}\right) \cos (\alpha x)\right],
$$

where $\bar{C}$ indicates a remaining constant. Another valid solution is provided by $\beta=\kappa^{2}$. In this case the transversal solution is given by

$$
G(y)=A \cdot \cos (\kappa \cdot y)
$$


Table 2: Uncoupled mode shapes sequence for the associated cavity.

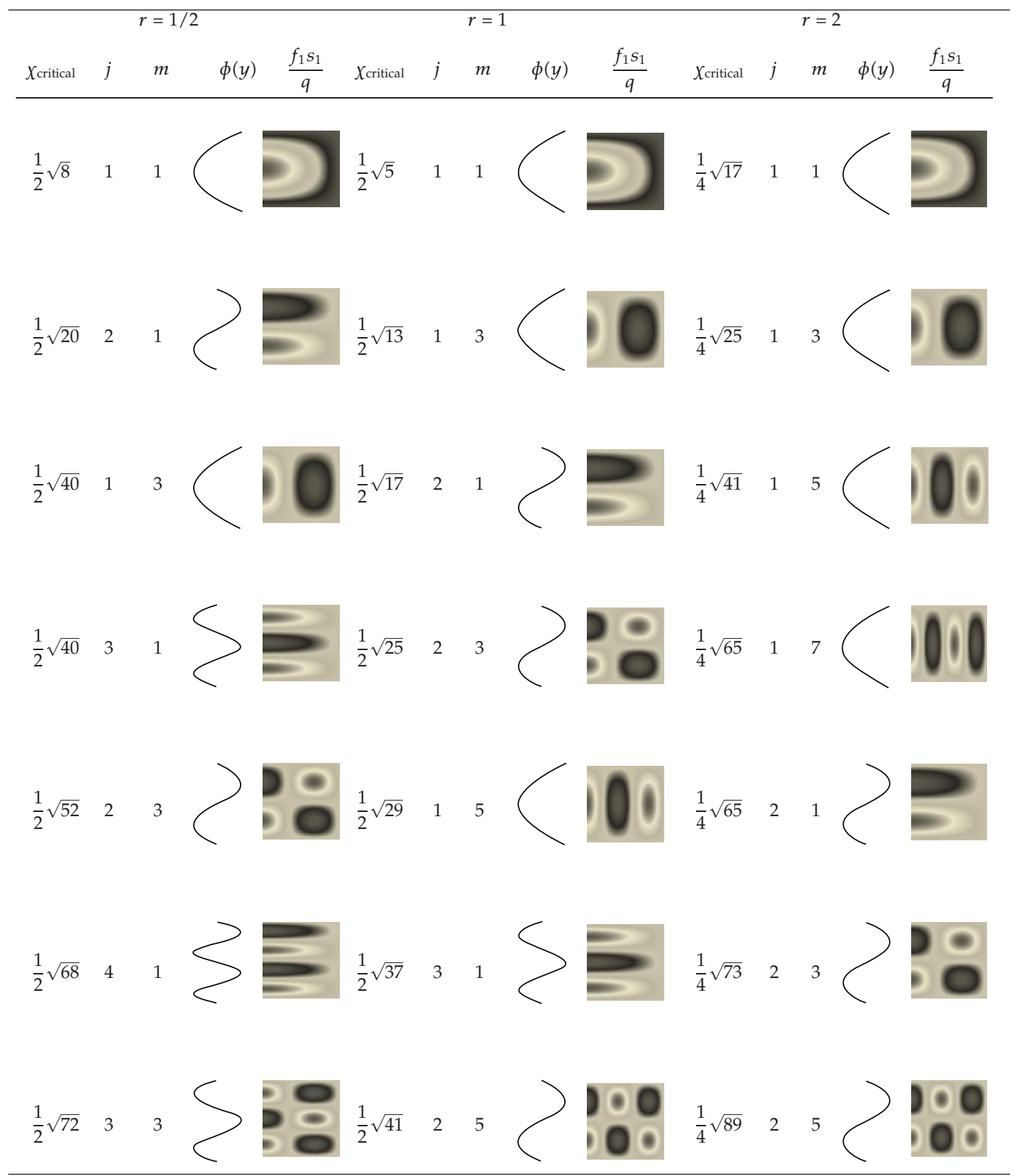

where

$$
\kappa=\frac{n \pi}{L_{y}} \quad n=0,1,2,3 \ldots
$$


It is important to notice that $\kappa=0$ in (3.25) provide a valid solution, given by (3.22). Therefore, solutions for $\beta=0$ are included in these last expressions, when $n=0$. The correspondent longitudinal solution is defined by

$$
F(x)=D \cdot\left[\sin (\sqrt{\alpha} \cdot x)-\tan \left(\sqrt{\alpha} \cdot L_{x}\right) \cdot \cos (\sqrt{\alpha} \cdot x)\right]
$$

where the separation constant $\alpha$ is defined by

$$
\alpha_{n}=\left(\frac{\omega}{c}\right)^{2}-\left(\frac{n \pi}{L_{y}}\right)^{2}, \quad n=0,1,2,3 \ldots
$$

The complete solution for $\beta=\kappa^{2}$ is given by

$$
P(x, y)=\sum_{n=0}^{\infty} E_{n} \cdot\left[\sin \left(\sqrt{\alpha_{n}} \cdot x\right)-\tan \left(\sqrt{\alpha_{n}} \cdot L_{x}\right) \cdot \cos \left(\sqrt{\alpha_{n}} \cdot x\right)\right] \cos \left(\kappa_{n} \cdot y\right)
$$

The remaining constant $E_{n}$ is obtained with application of boundary condition (3.3), using the cosine function orthogonality property. Thus,

$$
\begin{gathered}
E_{0}=-\frac{\rho_{f} \bar{A}}{L_{y} \sqrt{\alpha_{0}}} \int_{0}^{L_{y}} \phi(y) d y, \quad n=0, \\
E_{n}=-\frac{2 \rho_{f} \bar{A}}{L_{y} \sqrt{\alpha_{n}}} \int_{0}^{L_{y}} \phi(y) \cos \left(\kappa_{n} \cdot y\right) d y, \quad n=1,2,3 \ldots
\end{gathered}
$$

\section{Application of Sinusoidal Deformation Functions on S1 Boundary}

Assuming that the vibrating boundary $S 1$ is governed by (3.12) the following conditions are established by (3.30) integrals:

$$
\begin{aligned}
& \int_{0}^{L_{y}} \sin \left(\frac{j \pi y}{L_{y}}\right) d y=-\frac{L_{y}}{j \pi} \cdot[\cos (j \pi)-1]\left\{\begin{array}{l}
0 \longrightarrow j=\text { even, } \\
\frac{2 L_{y}}{j \pi} \longrightarrow j=\text { odd },
\end{array}\right. \\
& \int_{0}^{L_{y}} \sin \left(\frac{j \pi y}{L_{y}}\right) \cos \left(\frac{n \pi y}{L_{y}}\right) d y=L_{y} \cdot \frac{j[1-\cos (j \pi) \cos (n \pi)]}{\pi\left(j^{2}-n^{2}\right)}, \quad j \neq n .
\end{aligned}
$$

In expression (3.32) it must be observed that even values of $j$ result in nonzero solutions only for $n=1,3,5 \ldots$ If $j$ is an odd number, nontrivial solutions are expected only for $n=2,4,6 \ldots$ Therefore, it can be concluded that summation, indicated on (3.29), does not vanish on this 
case (probably the greatest disadvantage when compared to an entirely open cavity), with remaining values of $n$ providing valid solutions. Dynamic pressure solution is defined by

$$
\begin{aligned}
P_{0}\left(\xi_{x}\right)= & \frac{\rho_{f} \bar{A} L_{y}}{j \pi^{2}} \cdot \frac{\sin \left[\pi \chi\left(\xi_{x}-1\right) \cdot r\right] \cdot[\cos (j \pi)-1]}{x \cdot \cos (\pi x \cdot r)}, \quad n=0, \\
P_{n}\left(\xi_{x}, \xi_{y}\right)= & -\frac{2 \rho_{f} \bar{A} L_{y}}{\pi} \sum_{n=1}^{\infty} \frac{\sin \left[\pi \sqrt{x^{2}-n^{2}}\left(\xi_{x}-1\right) \cdot r\right]}{\sqrt{x^{2}-n^{2}} \cdot \cos \left(\pi \sqrt{x^{2}-n^{2}} \cdot r\right)} \\
& \cdot \frac{j[1-\cos (j \pi) \cos (n \pi)]}{\pi\left(j^{2}-n^{2}\right)} \cdot \cos \left(n \pi \xi_{y}\right), \quad n=1,2,3 \ldots
\end{aligned}
$$

The complete solution is given by sum of expressions (3.33) and (3.34). For even values of $j$ expression (3.33) vanishes and solution is defined only by (3.34), which assumes nonzero values for $n=1,3,5 \ldots$. For odd values of $j$ expressions (3.33) and (3.34) are present, with this last one assuming nontrivial solutions for $n=2,4,6 \ldots$

As in the previous case (entirely open cavity), critical values are also present in expressions (3.33) and (3.34), which are equivalent to the uncoupled cavity frequencies, with $S 1$ replaced by a rigid wall. These are defined by

$$
\chi_{\text {critical }}=\sqrt{\left(\frac{m}{2 r}\right)^{2}+n^{2}}, \quad n=0,1,2, \ldots, m=1,3,5 \ldots
$$

Limiting configurations (equivalent to the associated cavity modes) are established in expressions (3.33) and (3.34), when $\chi \rightarrow \chi_{\text {critical }}$. Table 3 presents these results for $r=1$, with corresponding values of $n$ and $m$. It is important to notice that odd values of $j$ are related to symmetrical distributions with respect to $\xi_{y}=1 / 2$. The opposite occurs for even values of $j$. Thus it can be concluded that symmetrical structural modes lead to symmetrical distributions of cavity pressures with respect to the vertical mid section.

\section{Fluid-Structure Coupled Solution for an Imposed Deformation}

The analyzed problem is illustrated on Figure 2. It consists of a general structure with a constant cross-section subjected to an external load. The dynamic response of this system can be represented by a generalized coordinate $X(t)$, allowing the construction of generalized parameters (mass, stiffness, and loading) for any arbitrary mode shape, related to $\phi(y)$. This type of solution will be very useful for the introduction of fluid pressures, since the previous developed approach for the fluid domain is also dependent on the shape function.

For the mathematical development of this problem the following considerations are assumed: mass per unit length $\mu(y)$, flexural rigidity $E I(y)$, length $L_{y}$, external distributed loading $F(y, t)$, and unitary width perpendicular to the $x y$ plane. The deflections are represented by $v(y, t)$, related to an arbitrary coordinate $X(t)$, and a mode shape function $\phi(y)$, normalized at the generalized coordinate location. Therefore,

$$
v(y, t)=\phi(y) X(t)
$$


Table 3: Uncoupled mode shapes sequence for the associated cavity $(r=1)$.

\begin{tabular}{|c|c|c|c|c|c|c|c|}
\hline \multirow[b]{2}{*}{$X_{\text {critical }}$} & \multicolumn{3}{|c|}{ Even values of $j$} & \multicolumn{4}{|c|}{ Odd values of $j$} \\
\hline & $n$ & $m$ & $\frac{f_{1} s_{1}}{q}$ & $X_{\text {critical }}$ & $n$ & $m$ & $\frac{f_{1} s_{1}}{q}$ \\
\hline$\frac{1}{2} \sqrt{5}$ & 1 & 1 & & $\frac{1}{2}$ & 0 & 1 & \\
\hline$\frac{1}{2} \sqrt{13}$ & 1 & 3 & & $\frac{3}{2}$ & 0 & 3 & \\
\hline$\frac{1}{2} \sqrt{29}$ & 1 & 5 & & $\frac{1}{2} \sqrt{17}$ & 2 & 1 & \\
\hline$\frac{1}{2} \sqrt{37}$ & 3 & 1 & & $\frac{5}{2}$ & 0 & 5 & \\
\hline$\frac{1}{2} \sqrt{53}$ & 1 & 7 & & $\frac{5}{2}$ & 2 & 3 & \\
\hline
\end{tabular}

The system's dynamic equilibrium equation is obtained with the virtual work principle, equaling the work done by internal and external forces. Thus,

$$
\int_{0}^{L_{y}} F(y, t) \phi(y) d y=\ddot{X} \int_{0}^{L_{y}} \mu(y)[\phi(y)]^{2} d y+X \int_{0}^{L_{y}} E I(y)\left[\frac{d^{2} \phi(y)}{d y^{2}}\right]^{2} d y .
$$

Finally, introducing the following notations:

$$
\begin{aligned}
& \widetilde{M}=\int_{0}^{L_{y}} \mu(y)[\phi(y)]^{2} d y, \\
& \widetilde{K}=\int_{0}^{L_{y}} E I(y)\left[\frac{d^{2} \phi(y)}{d y^{2}}\right]^{2} d y, \\
& \widetilde{F}=\int_{0}^{L_{y}} F(y, t) \phi(y) d y,
\end{aligned}
$$




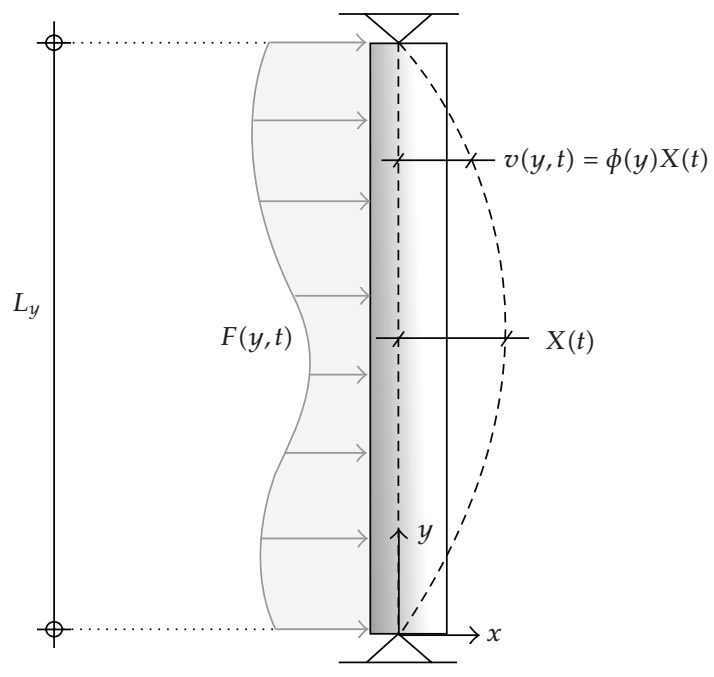

Figure 2: Representation scheme of the structural model.

and substitution of these last expressions in (4.2) provide

$$
\widetilde{M} \ddot{X}+\widetilde{K} X=\widetilde{F}(t),
$$

which represents the system's dynamic equilibrium equation of motion in terms of a generalized coordinate $X(t)$, where $\widetilde{M}, \widetilde{K}$, and $\widetilde{F}$ are related, respectively, to generalized mass, generalized stiffness, and generalized loading. It should be noticed that the generalized stiffness term includes only bending deformation effects. Additional contributions could be included by means of modification of this parameter. Damping could also be included, and in this case it would be more convenient to express this effect using a damping ratio $(\xi)$. Thus

$$
\widetilde{C}=2 \xi \widetilde{M} \omega .
$$

\subsection{Application of Fluid Pressures at the Interface}

The previous solution depends on an external loading. In the case of a coupled system this loading is represented by the dynamic pressures acting at the interface. Therefore

$$
F(y, t)=p(0, y, t) \times 1=P(0, y) e^{-i \omega t}
$$

where the function $P(0, y)$ is related to the corresponding cavity (associated to the framed structure), with boundary conditions depending on the analyzed solution for the fluid domain. For a harmonic vibrating contour accelerations at the interface are given by

$$
\ddot{u}(y, t)=\phi(y) \bar{A} e^{-i \omega t} .
$$


Accelerations at the interface are equivalent for both the structure and the fluid. Therefore,

$$
\ddot{u}(y, t)=\ddot{v}(y, t)=\phi(y) \ddot{X}(t) .
$$

Analysis of (4.9) and (4.10) provides

$$
\ddot{X}(t)=\bar{A} e^{-i \omega t} .
$$

Substitution of (4.5) and (4.8) in (4.6) gives

$$
\widetilde{M} \ddot{X}+\widetilde{K} X+e^{-i \omega t} \int_{0}^{L_{y}} P(0, y) \phi(y) d y=0 .
$$

In the above expression the generalized force is located at the left-hand side, because physically the dynamic pressure acts in the same direction of inertia and elastic forces. Equation (4.11) can be applied in (4.12) leading to a simplified expression given by

$$
\left[\widetilde{M}+\int_{0}^{L_{y}} \frac{P(0, y)}{\bar{A}} \phi(y) d y\right] \ddot{X}+\widetilde{K} X=\left[\widetilde{M}+\widetilde{M}_{\text {fluid }}\right] \ddot{X}+\widetilde{K} X=0 .
$$

Equation (4.13) represents the free vibration of the structural model, with a generalized mass produced by the interaction between fluid-solid domains. This expression can be simplified with the inclusion of a generalized added mass term, which corresponds to sum of the structural and fluid dislocated masses. Thus,

$$
\widetilde{M}_{\text {total }} \ddot{X}+\widetilde{K} X=0 \text {. }
$$

Expression (4.11) provides

$$
X(t)=-\frac{\bar{A} e^{-i \omega t}}{\omega^{2}} \longrightarrow \ddot{X}(t)=-\omega^{2} X(t) .
$$

Substitution of (4.15) in (4.14) results in

$$
\left(\tilde{K}-\omega^{2} \widetilde{M}_{\text {total }}\right) X=0 .
$$

For a nontrivial solution the term in brackets of (4.16) must be null. Therefore,

$$
\widetilde{K}-\omega^{2} \widetilde{M}_{\text {total }}=0 .
$$

Solution of the above expression provides frequencies of the coupled problem. It should be noticed that the generalized stiffness depends on $\phi(y)$. And the total generalized mass is composed of two parts. The first one is structure related, being dependent on $\phi(y)$. The 
second one is associated to the fluid dislocated mass and depends on $\phi(y)$ and $\omega$, which are unknown parameters of the problem, corresponding to the structural coupled mode shape and the coupled system frequency, respectively. Therefore, this type of solution establishes only one equation and two unknown variables. A simplified solution is possible with the introduction of an imposed deformation function at the interface. Thus, a frequency equation for a corresponding mode shape is constructed and the corresponding set of solutions is obtained. Latter these values can be applied in the total generalized added mass expression, resulting in the system's dynamic equilibrium equation of motion, which can be solved for an arbitrary excitation. Or, they can be substituted in the pressure field solution, resulting in the cavity coupled mode shapes.

\subsection{Frequency Equation for an Open Cavity with a Sinusoidal Vibrating Boundary}

In this specific case fluid pressure solution is given by (3.15). At the interface $\xi_{x}=0$ of a square cavity $(r=1)$ this expression is reduced to

$$
P\left(0, \xi_{y}\right)=\frac{\rho_{f} \bar{A} L_{y}}{\pi} \cdot \frac{\tan \left(\pi \sqrt{x^{2}-j^{2}}\right)}{\sqrt{x^{2}-j^{2}}} \cdot \sin \left(j \pi \xi_{y}\right) .
$$

Thus, the generalized added mass is given by

$$
\widetilde{M}_{\text {fluid }}=\int_{0}^{L_{y}} \frac{P\left(0, \xi_{y}\right)}{\bar{A}} \phi(y) d y=\frac{\rho_{f} L_{y}{ }^{2}}{2 \pi} \cdot \frac{\tan \left(\pi \sqrt{x^{2}-j^{2}}\right)}{\sqrt{x^{2}-j^{2}}} .
$$

This last equation represents the generalized fluid-added mass solution for a square open cavity with a harmonic vibrating boundary associated to $\phi(y)=\sin \left(j \pi y / L_{y}\right)$. Therefore, the dynamic equilibrium equation of this system is given by substitution of (4.19) in (4.17):

$$
\tilde{K}-\omega^{2}\left[\widetilde{M}+\frac{\rho_{f} L_{y}^{2}}{2 \pi} \cdot \frac{\tan \left(\pi \sqrt{x^{2}-j^{2}}\right)}{\sqrt{x^{2}-j^{2}}}\right]=0,
$$

where the generalized parameters $\widetilde{K}$ and $\widetilde{M}$ are given by

$$
\begin{gathered}
\tilde{K}=E I\left(\frac{j \pi}{L_{y}}\right) \int_{0}^{L}\left[\cos \left(\frac{j \pi y}{L_{y}}\right)\right]^{2} d y=E I \frac{(j \pi)^{4}}{2 L_{y}{ }^{3}}, \\
\widetilde{M}=\mu \int_{0}^{L}\left[\sin \left(\frac{j \pi y}{L_{y}}\right)\right]^{2} d y=\frac{\mu L_{y}}{2} .
\end{gathered}
$$


Table 4: Values of $f(x, j)$ function for $x \rightarrow 0$.

\begin{tabular}{cccccc}
\hline & \multicolumn{5}{c}{$j$} \\
& 1 & 2 & 3 & 4 & 5 \\
\hline $\tanh (\pi j) / j$ & 0.9963 & 0.5000 & 0.3333 & 0.2500 & 0.2000 \\
\hline
\end{tabular}

Substitution of (4.21) in (4.20) provides

$$
E I \frac{(j \pi)^{4}}{2 L_{y}^{3}}-\omega^{2}\left[\frac{\mu L_{y}}{2}+\frac{\rho_{f} L_{y}^{2}}{2 \pi} \cdot \frac{\tan \left(\pi \sqrt{x^{2}-j^{2}}\right)}{\sqrt{x^{2}-j^{2}}}\right]=0
$$

which indicates the frequency equation of the coupled problem. For the uncoupled case the second term in brackets vanishes and the corresponding solution is given by

$$
\omega_{\text {vacuum }}^{j}=(j \pi)^{2} \sqrt{\frac{E I}{\mu L_{y}{ }^{4}}} .
$$

For the coupled case, solution of (4.22) is more complicated and includes infinite solutions for a given value of $j$ (as it will be demonstrated later). The second term in brackets is a function of $x$, and it is interesting to study the variation of this term with this parameter, which can be simplified to

$$
\widetilde{M}_{\text {fluid }}=\frac{\rho_{f} L_{y}^{2}}{2 \pi} \cdot f(x, j) \text {. }
$$

Figure 3 illustrates the variation of (4.24) along the $x$ axis for values of $j=1$ and 3 . Analysis of this graphic indicates that the generalized fluid-added mass solution is hyperbolic and without critical values (resonances) in the $x<j$ interval. Occurrences of critical points are expected beyond this point, leading the generalized added mass to infinite values (due to the trigonometric nature of the solution). It is interesting to notice that values of $x \ll j$ produce almost constant functions, with defined values at $x=0$.

The limit when $x \rightarrow 0$ establishes

$$
\lim _{x \rightarrow 0} \widetilde{M}_{\text {fluid }}=\frac{\rho_{f} L_{y}^{2}}{2 \pi} \cdot \frac{\tanh (\pi j)}{j}
$$

which indicates a generalized fluid-added mass solution independent of $\omega$. Values of this function can be evaluated for a given value of $j$. Table 4 illustrates these results. Reduced values of the added mass are expected with the increasing of $j$. 


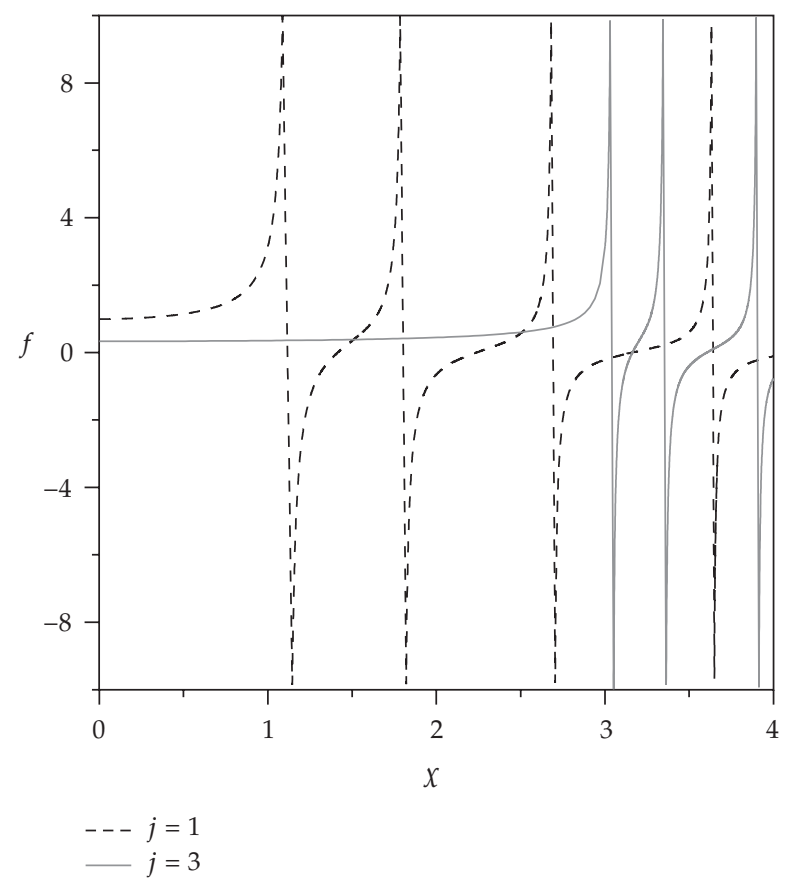

Figure 3: Variation of $f(x, j)$ along the $x$ axis for values of $j=1$ and 3 .

It is also possible to rewrite (4.22) in terms of the uncoupled frequencies of both the structure and the associated cavity. Thus,

$$
\frac{\mu L_{y}}{2}\left\{\left(\omega_{\text {vacuum }}^{j}\right)^{2}-\left(x \omega_{\text {cavity }}^{1}\right)^{2}\left[1+\frac{\rho_{f} L_{y}}{\mu \pi} \cdot \frac{\tan \left(\pi \sqrt{x^{2}-j^{2}}\right)}{\sqrt{x^{2}-j^{2}}}\right]\right\}=0,
$$

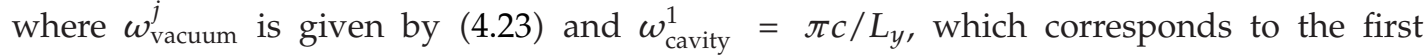
transversal frequency of the uncoupled cavity. Solutions of (4.26) are established when the term in curly brackets vanishes. Therefore,

$$
\left(\mathscr{F}_{1}\right)^{2}-x^{2}\left[1+\frac{\mathscr{F}_{2}}{\pi} \cdot \frac{\tan \left(\pi \sqrt{x^{2}-j^{2}}\right)}{\sqrt{x^{2}-j^{2}}}\right]=J 1-J 2=0,
$$

where the following dimensionless parameters are defined: $\mathcal{F}_{1}=\omega_{\text {vacuum }}^{j} / \omega_{\text {cavity }}^{1}$ and $\mathcal{F}_{2}=$ $\rho_{f} L_{y} / \mu$. This last term can be rewritten, resulting in

$$
\mathcal{F}_{2}=\frac{\rho_{f} \cdot L_{y}}{\rho_{s} \cdot e \cdot 1}=\frac{\bar{\rho}}{\mathcal{R}}
$$




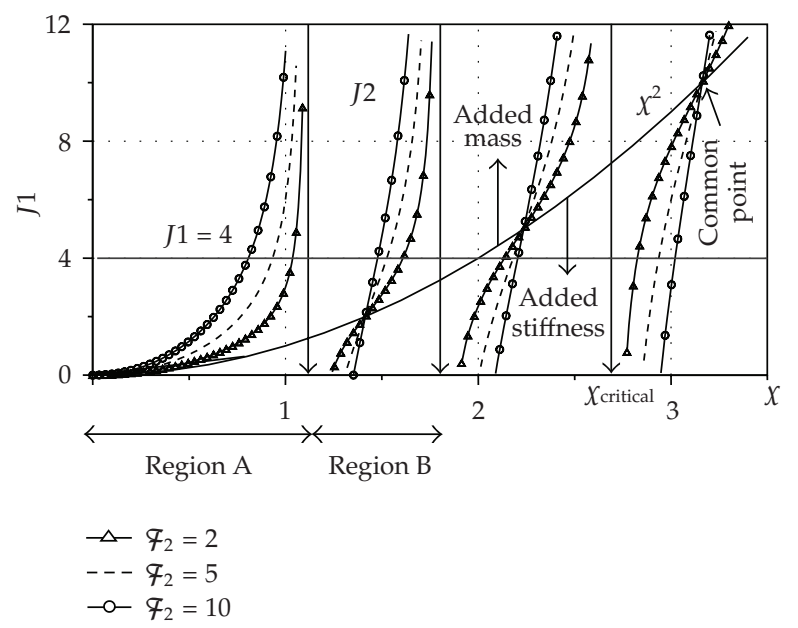

Figure 4: Parametric abacus of the frequency equation for $j=1$ and $r=1$.

with $\bar{\rho}=\rho_{f} / \rho_{s}$ and $R=e / L_{y}$. Therefore, the coupled solutions are defined in terms of four parameters: the structural frequency of the corresponding mode shape in vacuum, the first transversal frequency of the uncoupled cavity, the density relation between fluid and structure, and the thickness/height ratio of the structure.

A parametric study of (4.27) is presented on Figure 4, for $j=1$. The distributed plots are associated to function $J 2$, which represents the second term in this expression. The coupled values of $x$ are established at the intersection of these curves with a corresponding horizontal line, which indicates the value of $J 1$. Constant values of $J 1=4$ are illustrated on this figure, providing a reference for analysis of the involved parameters.

Analysis of Figure 4 indicates the presence of two distinct regions: A and B. The first one is contained on the initial interval between $x=0$ and the first critical value (first resonance). The behavior of $J 2$ function at this region is given by curves with developing amplitudes towards infinity. The second region is composed by curves with amplitudes ranging from $-\infty$ to $+\infty$. In a given point there is a common intersection, where all the functions share the same amplitude. In both regions an increase of $\mathcal{F}_{2}$ results in greater horizontal distances between $J 2$ function and the vertical asymptotes (corresponding to the cavity resonance frequencies). This implies greater relative differences between the coupled problem solution and the corresponding uncoupled cavity frequencies. It should also be noticed that an arbitrary horizontal line $(J 1=4$, e.g.) will provide infinite solutions, intercepting $J 2$ curves more than once.

Limit zones are established by the solid curve $x^{2}$, which connects the common intersection points in Region B. This condition implies $J 1=\chi^{2}$, leading to

$$
\left(\frac{\omega_{\text {vacuum }}^{j}}{\omega_{\text {cavity }}^{1}}\right)^{2}=\left(\frac{\omega}{\omega_{\text {cavity }}^{1}}\right)^{2} \therefore \omega=\omega_{\text {vacuum }}^{j} .
$$

Thus, solutions intercepted by $x^{2}$ curve will present coupled frequencies which are equal to the corresponding in-vacuum values. Additionally, this function defines solution zones of added mass and added stiffness, which are located, respectively, above and below this curve. 
An added mass region implies coupled frequencies inferior to the corresponding in-vacuum values, while an added stiffness implies the opposite. It should be noted that all solutions in Region A are of added mass. Therefore, the initial solution will always be of this type. For Region B both types of solutions are possible, including the common intersection points. Solutions in Region A are unique, while for Region B an infinite set of solutions is defined. The common points in Region B are given by

$$
-\frac{\mp_{2}}{\pi} \cdot \frac{\tan \left(\pi \sqrt{x^{2}-j^{2}}\right)}{\sqrt{x^{2}-j^{2}}}=0 .
$$

Solution of this last expression results in

$$
\pi \sqrt{x^{2}-j^{2}}=\bar{m} \pi, \quad \bar{m}=1,2,3 \ldots
$$

Therefore,

$$
x=\sqrt{\bar{m}^{2}+j^{2}}, \quad \bar{m}=1,2,3 \ldots
$$

which provides the common points solution, which is independent of $\mathcal{F}_{2}$. The corresponding solution of $J 1$ when condition (4.29) is established is given by

$$
J 1=x^{2} \therefore J 1=\bar{m}^{2}+j^{2} .
$$

Therefore, problems with condition (4.33) satisfied will present a single coupled frequency which is equal to the corresponding in-vacuum value. As mentioned before, this type of solution is always located on Region B.

Figures 5 and 6 present, respectively, the parametric abacuses for $j=2$ and $j=3$. In both cases Region A is extended to the proximity of $x=j$. Observations made for Figure 4 are still valid.

It is important to notice that previous analyses were concerned with square cavities $(r=1)$. However, the same procedure could be extended to a rectangular geometry. In this case the generalized added mass will be dependent on the $r$ parameter. Therefore, (4.18) can be replaced by

$$
P\left(0, \xi_{y}\right)=\frac{\rho_{f} \bar{A} L_{y}}{\pi} \cdot \frac{\tan \left(r \pi \sqrt{x^{2}-j^{2}}\right)}{\sqrt{x^{2}-j^{2}}} \cdot \sin \left(j \pi \xi_{y}\right) .
$$

The generalized added mass resulting from this last expression is given by

$$
\widetilde{M}_{\text {fluid }}=\frac{\rho_{f} L_{y}^{2}}{2 \pi} \cdot \frac{\tan \left(r \pi \sqrt{x^{2}-j^{2}}\right)}{\sqrt{x^{2}-j^{2}}}=\frac{\rho_{f} L_{y}^{2}}{2 \pi} \cdot f(r, x, j) .
$$




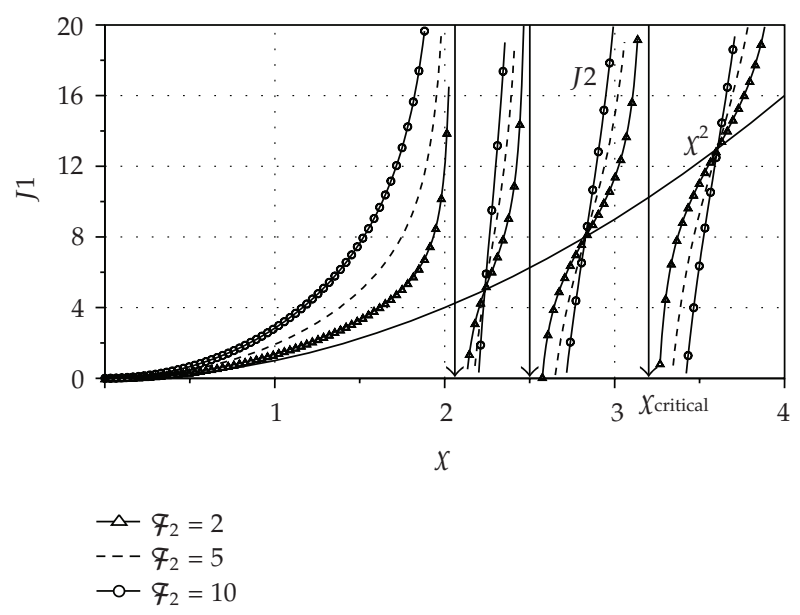

Figure 5: Parametric abacus of the frequency equation for $j=2$ and $r=1$.

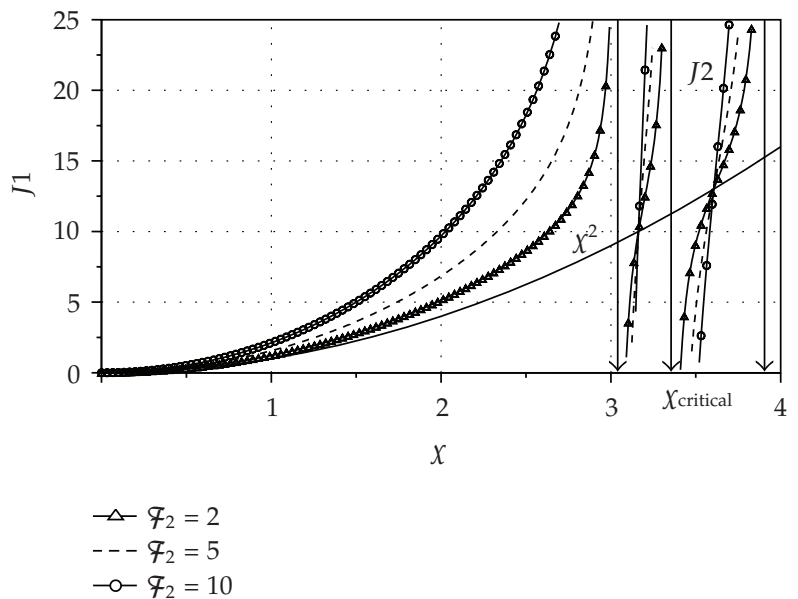

Figure 6: Parametric abacus of the frequency equation for $j=3$ and $r=1$.

Thus, the corresponding frequency equation results in

$$
\left(\mp_{1}\right)^{2}-x^{2}\left[1+\frac{\mp_{2}}{\pi} \cdot \frac{\tan \left(r \pi \sqrt{x^{2}-j^{2}}\right)}{\sqrt{x^{2}-j^{2}}}\right]=J 1-J 2=0 .
$$

The effects of $r$ in the parametric abacuses are related to the resonance values given by (3.18). An increasing value of this parameter results in a greater number of critical points at a given interval along the $x$ axis, resulting in more regions of type B (Figure 7). The first resonance is also influenced by this parameter, defining the horizontal extension of Region A. Decreasing the value of $r$ will result in a larger extension of Region A, and in a smaller number of regions of type B (Figure 8). 


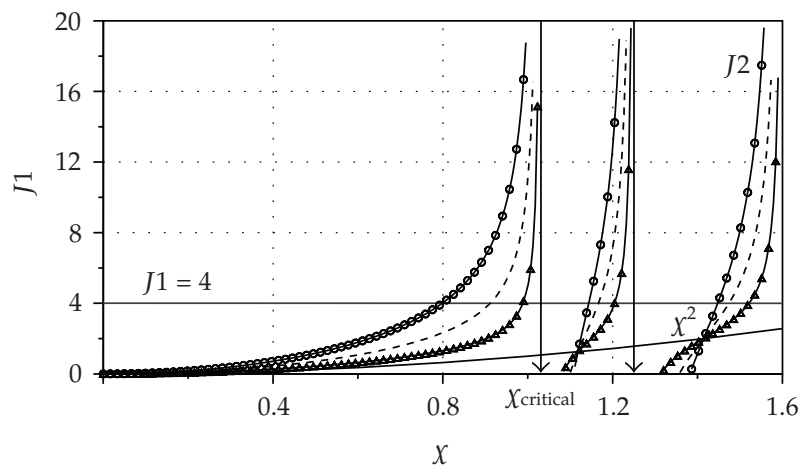

$$
\begin{aligned}
& -\boldsymbol{f}_{2}=2 \\
& --\boldsymbol{f}_{2}=5 \\
& --\boldsymbol{f}_{2}=10
\end{aligned}
$$

Figure 7: Parametric abacus of the frequency equation for $j=1$ and $r=2$.

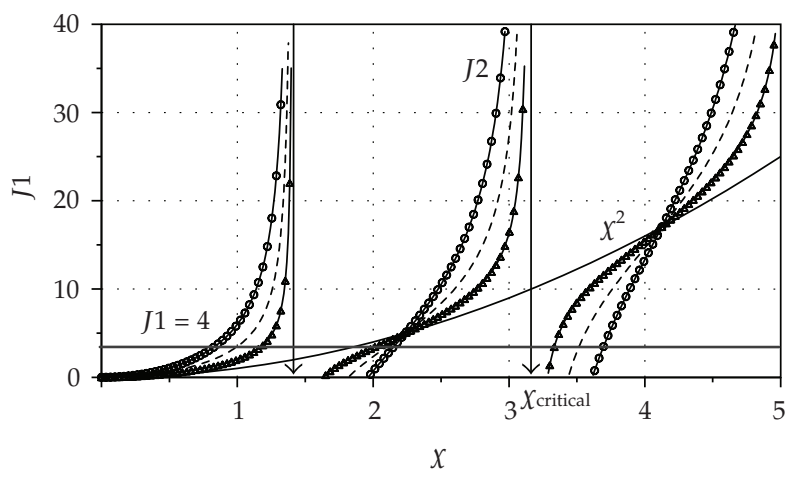

$$
\begin{aligned}
& -\triangle \mathcal{F}_{2}=2 \\
& --\mathcal{F}_{2}=5 \\
& \multimap-\mathcal{F}_{2}=10
\end{aligned}
$$

Figure 8: Parametric abacus of the frequency equation for $j=1$ and $r=1 / 2$.

The limit of expression (4.35) when $x \rightarrow 0$ is given by

$$
\lim _{x \rightarrow 0} \widetilde{M}_{\text {fluid }}=\frac{\rho_{f} L_{y}^{2}}{2 \pi} \cdot \frac{\tanh (r \pi j)}{j} .
$$

Equation (4.37) can be evaluated for given values of $j$ and $r$. Table 5 illustrates these results. Reduced values of the added mass are obtained for $r<1$ (short cavities). However, these effects are stronger at smaller values of $j$, exerting little influence on higher modes. Cavities with $r \geq 1$ (long cavities) present the same added mass for a given value of $j$.

Figures 7 and 8 illustrate, respectively, the effects of $r$ in the parametric abacuses for $j=1$. Comparison with Figure 4 indicates that regions of type B are developed earlier for $r=2$, with the second region of this type appearing before $X=1.6$. The opposite occurs for $r=1 / 2$, with these regions dislocated to higher values of $x$. 
Table 5: Values of $f(r, x, j)$ function for $x \rightarrow 0$.

\begin{tabular}{lccccc}
\hline & \multicolumn{5}{c}{$j$} \\
& 1 & 2 & 3 & 4 & 5 \\
\hline$r=1 / 4$ & 0.6558 & 0.4586 & 0.3274 & 0.2491 & 0.1998 \\
$r=1 / 2$ & 0.9172 & 0.4981 & 0.3333 & 0.2500 & 0.2000 \\
$r \geq 1$ & 0.9963 & 0.5000 & 0.3333 & 0.2500 & 0.2000 \\
\hline
\end{tabular}

Table 6: Generalized fluid-added mass conditions.

\begin{tabular}{lccc}
\hline value of $j$ & $n$ & $\widetilde{M}_{\text {fluid 1 }}$ & $\widetilde{M}_{\text {fluid 2 }}$ \\
\hline even & $1,3,5 \ldots$ & null & defined \\
odd & $2,4,6 \ldots$ & defined & defined \\
\hline
\end{tabular}

\subsection{Frequency Equation for a Closed Cavity in the Transversal Direction with a Sinusoidal Vibrating Boundary}

In this specific case fluid pressure solution is given by (3.33) and (3.34). At the interface $\xi_{x}=0$ of a square cavity $(r=1)$ these expressions are reduced to

$$
\begin{gathered}
P_{0}(0)=-\frac{\rho_{f} \bar{A} L_{y}}{j \pi^{2}} \cdot \frac{\tan (\pi \chi) \cdot[\cos (j \pi)-1]}{\chi}, \\
P_{n}\left(0, \xi_{y}\right)=\frac{2 \rho_{f} \bar{A} L_{y}}{\pi} \sum_{n=1}^{\infty} \frac{\tan \left(\pi \sqrt{\chi^{2}-n^{2}}\right)}{\sqrt{x^{2}-n^{2}}} \cdot \frac{j[1-\cos (j \pi) \cos (n \pi)]}{\pi\left(j^{2}-n^{2}\right)} \cdot \cos \left(n \pi \xi_{y}\right) .
\end{gathered}
$$

The generalized added mass is given by

$$
\widetilde{M}_{\text {fluid }}=\int_{0}^{L_{y}} \frac{P_{0}(0)}{\bar{A}} \phi(y) d y+\int_{0}^{L_{y}} \frac{P_{n}\left(0, \xi_{y}\right)}{\bar{A}} \phi(y) d y=\widetilde{M}_{\text {fluid } 1}+\widetilde{M}_{\text {fluid } 2 .}
$$

This last expression can be evaluated using orthogonality properties of sine and cosine functions. Therefore, substituting (4.38) in (4.39)

$$
\begin{aligned}
& \widetilde{M}_{\text {fluid } 1}=\frac{\rho_{f} L_{y}^{2}}{\pi^{3}} \cdot \frac{\tan (\pi \chi)}{\chi} \cdot \frac{[\cos (j \pi)-1]^{2}}{j^{2}}, \\
& \widetilde{M}_{\text {fluid 2 }}=\frac{2 \rho_{f} L_{y}^{2}}{\pi^{3}} \sum_{n=1}^{\infty} \frac{\tan \left(\pi \sqrt{x^{2}-n^{2}}\right)}{\sqrt{x^{2}-n^{2}}} \cdot\left[\frac{j[1-\cos (j \pi) \cos (n \pi)]}{\left(j^{2}-n^{2}\right)}\right]^{2} .
\end{aligned}
$$

It should be noticed that conditions related to dynamic pressures are still valid for these generalized added masses. Thus, expression (4.40) vanishes for even values of $j$, with (4.41) resulting in non trivial solutions for odd values of $n$. Table 6 presents these conclusions. 


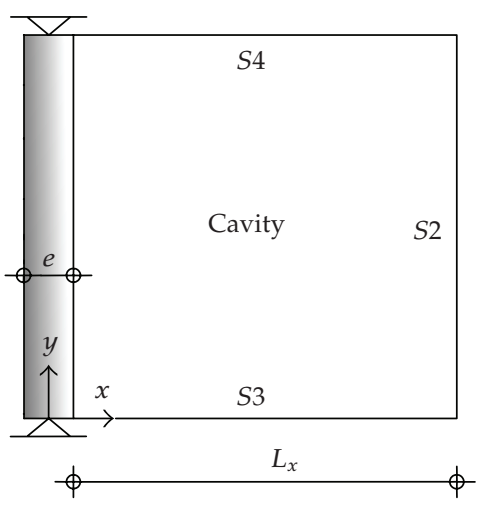

(a)

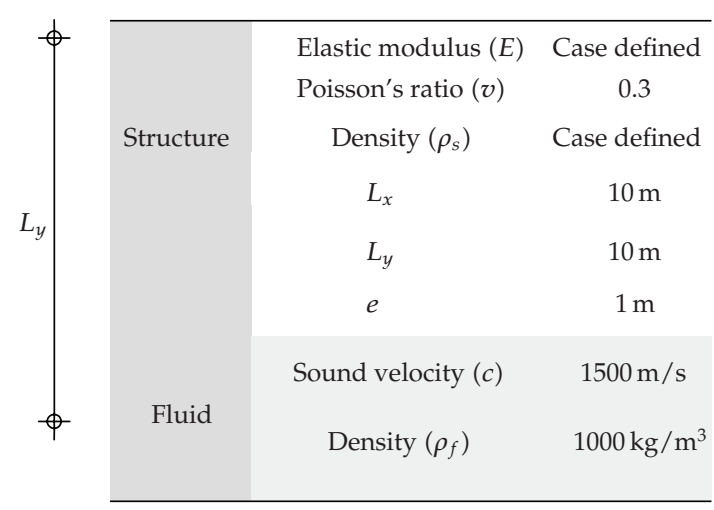

(b)

Figure 9: General analysis scheme, material and geometrical properties.

As in the previous case, a frequency equation is established by

$$
\left(\mathscr{F}_{1}\right)^{2}-x^{2}\left[1+\frac{\mathscr{F}_{2}}{\pi^{3}} \cdot \mathcal{M}\right]=J 1-J 2=0
$$

with $\mathcal{M}$ defining the following parameter:

$$
\mathcal{M}=\frac{2 \pi^{3}}{\rho_{f} L_{y}^{2}} \widetilde{M}_{\text {fluid }}=\frac{2 \pi^{3}}{\rho_{f} L_{y}^{2}}\left(\widetilde{M}_{\text {fluid } 1}+\widetilde{M}_{\text {fluid 2 }}\right) .
$$

\section{Application Examples and Results}

Two application examples of the previous described procedures are presented on this item. Simple beams associated to acoustic cavities entirely open (1) and closed in the transversal direction (2) are solved analytically and these results are compared to a finite element analysis. Figure 9 illustrates the general analysis scheme with material and geometrical properties.

\subsection{Analysis 1-Entirely Open Cavity $(p=0 \rightarrow S 2, S 3, S 4)$}

In this specific case the structural elastic modulus and density are taken, respectively, as $E=$ $2.1 \cdot 10^{11} \mathrm{~N} / \mathrm{m}^{2}$ and $\rho_{s}=7800 \mathrm{~kg} / \mathrm{m}^{3}$. Parameters $\mathcal{F}_{1}$ and $\mathcal{F}_{2}$ are given by

$$
\begin{aligned}
& \mathcal{F}_{1}=\frac{\omega_{\text {vacuum }}^{j}}{\omega_{\text {cavity }}^{1}}, \\
& \mathcal{F}_{2}=\frac{1000 \cdot 10}{7800 \cdot 1 \cdot 1} \cong 1.28 .
\end{aligned}
$$


Therefore, the above equations indicate that $\mathcal{F}_{2}$ parameter is constant, while $\mathcal{F}_{1}$ depends on $j$, which is associated to the structural vibration mode. The first three structural in-vacuum modes have the following frequencies:

$$
\omega_{\text {vacuum }}^{1} \cong 147.83 \mathrm{rad} / \mathrm{s} ; \quad \omega_{\text {vacuum }}^{2} \cong 591.33 \mathrm{rad} / \mathrm{s} ; \quad \omega_{\text {vacuum }}^{3} \cong 1330.50 \mathrm{rad} / \mathrm{s}
$$

The first transversal frequency of the uncoupled cavity is given by

$$
\omega_{\text {cavity }}^{1}=\frac{\pi c}{L_{y}} \cong 471.24 \mathrm{rad} / \mathrm{s}
$$

Thus, for the first three modes $\mathcal{F}_{1}^{j}$ parameter presents the following values:

$$
\mathcal{F}_{1}^{1} \cong 0.31 ; \quad \mathcal{F}_{1}^{2} \cong 1.25 ; \quad \boldsymbol{F}_{1}^{3} \cong 2.82
$$

The above values together with (5.2) can be applied in the parametric abacuses corresponding to the associated value of $j$, or substituted in (4.27) for solution of the coupled values of $x$ in a given mode shape. Coupled frequencies are given by

$$
\omega=\frac{\chi \pi c}{L_{y}}
$$

Table 7 illustrates hydrodynamic pressure distribution, coupled frequencies, and boundary deformation associated to the first seven vibration modes, obtained analytically and numerically using ANSYS finite element code. The last two columns on the right side indicate, respectively, frequencies and mode shapes of the uncoupled cavity (which are equal to results presented on Table 2 for $r=1$ ). An alternative type of representation of these results is illustrated on Table 8 , which presents the relative differences between coupled analytical and numerical frequencies, as well as the relative differences between analytical coupled and uncoupled cavity frequencies.

Analysis of Tables 7 and 8 results indicates that modes 1, 2, and 6 do not present characteristics of cavity modes (or resonant modes). The remaining coupled modes demonstrate certain proximity with the corresponding cavity frequencies and mode shapes, presenting modes slightly dislocated in the horizontal direction when compared to the cavity solution. In these cases the relative differences between coupled frequencies and cavity values are inferior to $10 \%$. Modes 1, 2, and 6 have coupled frequencies inferior to the corresponding in-vacuum values. Thus, it can be concluded that these modes are included in the added mass region of the abacuses (above the $x^{2}$ curve).

An alternative approach could be used for solution of coupled frequencies of modes 1, 2, and 6. Application of Table 5 and (4.37) provides approximate values of the generalized fluid-added mass, for solution of coupled frequencies located in Region A of the parametric abacuses. Therefore, coupled frequencies are given by

$$
\omega=\sqrt{\frac{\tilde{K}}{\widetilde{M}_{\text {total }}}}=\sqrt{\frac{\tilde{K}}{\widetilde{M}+\widetilde{M}_{\text {fluid }}}} .
$$


Table 7: Analytical and numerical solutions (Analysis 1).

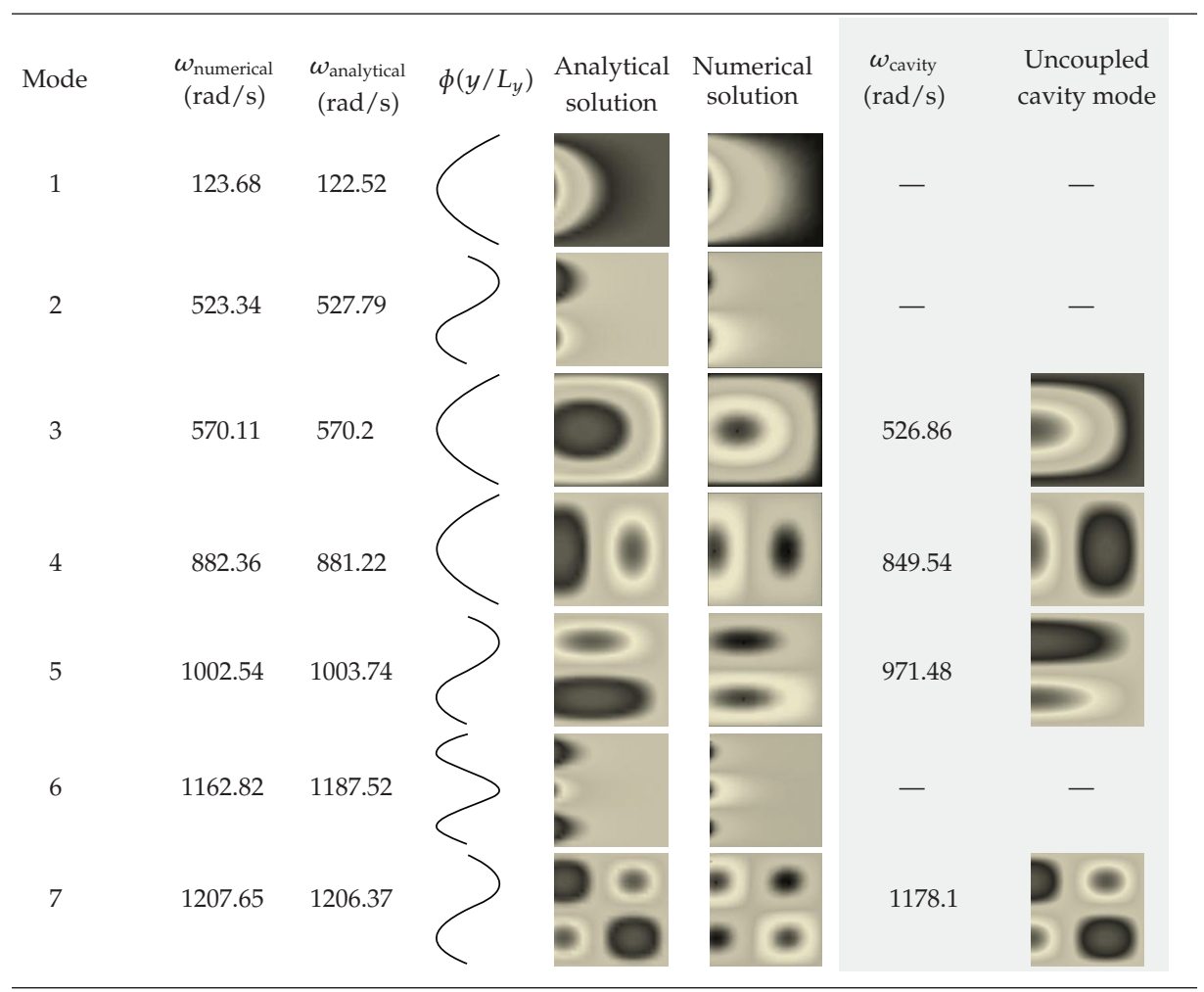

Table 8: Relative differences between analyzed frequencies (Analysis 01).

\begin{tabular}{|c|c|c|}
\hline \multirow{2}{*}{ Mode } & $\left|\omega_{\text {numerical }}-\omega_{\text {analytical }}\right| \cdot 100$ & $\left|\omega_{\text {analytical }}-\omega_{\text {cavity }}\right| \cdot 100$ \\
\hline & $\omega_{\text {numerical }}$ & $\omega_{\text {cavity }}$ \\
\hline 1 & 0.94 & - \\
\hline 2 & 0.85 & - \\
\hline 3 & 0.02 & 8.23 \\
\hline 4 & 0.13 & 3.73 \\
\hline 5 & 0.12 & 3.32 \\
\hline 6 & 2.12 & - \\
\hline 7 & 0.11 & 2.40 \\
\hline
\end{tabular}

Thus,

$$
\omega_{\text {coupled }}^{1} \cong 122.52 \mathrm{rad} / \mathrm{s} ; \quad \omega_{\text {coupled }}^{2} \cong 537.21 \mathrm{rad} / \mathrm{s} ; \quad \omega_{\text {coupled }}^{3} \cong 1248.78 \mathrm{rad} / \mathrm{s} .
$$

This presents an excellent agreement with corresponding values indicated on Table 7. It should be noticed that better results are expected for $x \ll j$. Therefore, solution for $j=$ $1(x=0.26)$ is almost exact, while for $j=3(x=2.65)$ an error of $7 \%$ is encountered. 


\subsection{Analysis 2-Closed Cavity in the Transversal Direction$$
(p=0 \rightarrow S 2 ; \partial p / \partial y=0 \rightarrow S 3, S 4)
$$

In this specific case the structural elastic modulus and density are taken, respectively, as $E=$ $2.5 \cdot 10^{10} \mathrm{~N} / \mathrm{m}^{2}$ and $\rho_{s}=2000 \mathrm{~kg} / \mathrm{m}^{3}$. Parameter $\mathcal{F}_{2}$ is given by

$$
\mathcal{F}_{2}=\frac{1000 \cdot 10}{2000 \cdot 1 \cdot 1}=5
$$

The first three structural in-vacuum modes have the following frequencies:

$$
\omega_{\text {vacuum }}^{1} \cong 100.73 \mathrm{rad} / \mathrm{s} ; \quad \omega_{\text {vacuum }}^{2} \cong 402.92 \mathrm{rad} / \mathrm{s} ; \quad \omega_{\text {vacuum }}^{3} \cong 906.58 \mathrm{rad} / \mathrm{s} .
$$

The corresponding values of $\Psi_{1}^{j}$ parameter are given by

$$
\mathcal{F}_{1}^{1} \cong 0.21 ; \quad \mathcal{F}_{1}^{2} \cong 0.86 ; \quad \mathcal{F}_{1}^{3} \cong 1.92
$$

Table 9 illustrates hydrodynamic pressure distribution, coupled frequencies, and boundary deformation associated to the first seven vibration modes, obtained analytically and numerically using ANSYS finite element code. Modes 1, 2, and 5 have coupled frequencies smaller than the corresponding in-vacuum values, indicating fluid-added mass effects. Modes 2, 4, and 7 demonstrate certain proximity with cavity results, with configurations slightly dislocated in the horizontal direction. In the remaining modes, where $j$ is an odd number, this observation is not very clear. Solutions of this type include cavity modes associated to null values of $n$. Therefore, modes 1 and 3 are related to the first cavity resonance $(m=1, n=0)$, while modes 5 and 6 are related to the third cavity mode $(m=3, n=0)$.

It is important to notice that in this case fluid effects can modify structural in-vacuum mode shapes, as it can be noticed on modes 3,6 , and 7 . The proposed procedure remains valid if a dominant configuration is still given by simple beam mode shapes, such as in modes 3 and 7. However, mode 6 presents an exception, where no dominant configuration is identified.

\subsection{Results Discussion for Analysis 1}

The previous results indicate the division of coupled modes in two distinct categories. In the first type, coupled frequencies and mode configurations are very different from the corresponding uncoupled cavity solutions (illustrated on Table 2). In the second type, coupled modes show great resemblance with the uncoupled cavity configurations, with solutions dislocated in the horizontal direction and with a certain proximity in frequency values (which depends on both $\mathcal{F}_{1}$ and $\mathcal{F}_{2}$, with smaller differences expected for solutions of $x$ located near the resonances). The first type has characteristics of added mass, with coupled frequencies smaller than the corresponding structural in-vacuum values. Solutions of this type are located on Region A in the parametric abacuses. The second type presents the possibility of coupled frequencies smaller (added mass), greater (added stiffness), or even equal to the corresponding structural in-vacuum solutions. Solutions of this type are always located on Region B. 
Table 9: Analytical and numerical solutions (Analysis 2).

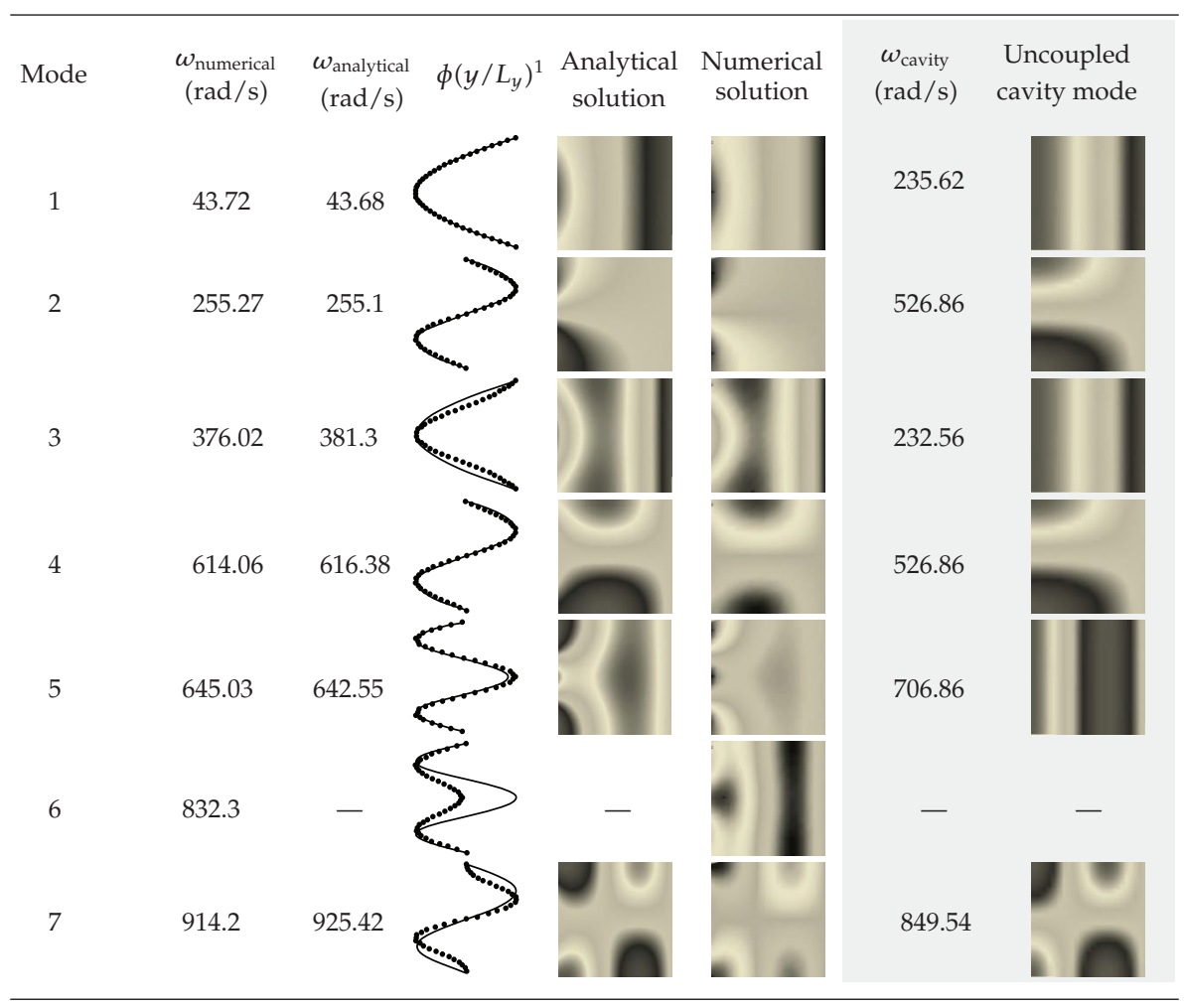

numerical (coupled); -analytical (in-vacuum).

An important conclusion can be established based on the previous results. A given mode $j$, located on Region A, with hyperbolic solution, will present $x_{j}$ given by

$$
x_{j}<j \therefore \omega_{j}<\frac{j \pi c}{L_{y}}
$$

From this last expression it is possible to conclude that structural modes with coupled frequencies inferior to the right side of (5.12) provide typical added mass solutions. That is, coupled modes were the structure exerting higher influence in the response. It is also important to notice that this limit is given by the uncoupled cavity frequencies in the transverse direction.

Typical added mass solutions are given by the modified first cavity resonance for a given $j$, with a hyperbolic solution in the longitudinal direction. Therefore, for these modes it is expected a decay in the dynamic pressure solution towards $x=L_{x}$. Table 10 illustrates the possible configurations for typical added mass modes related to $j=1$ to 3 .

Configurations of the coupled modes can be divided into distinct regions, according to the value of $x_{j}$. Values of $x_{j}<j$ define hyperbolic variations, with the resulting solution located before the first resonance. Solutions in this region are classified as typical added mass modes (Region I). Values of $j<x_{j}<x_{\text {critical }}^{1}$ define trigonometric solutions, with the corresponding mode shape resembling the first resonance configuration slightly dislocated 
Table 10: Possible configurations for the typical added mass modes.

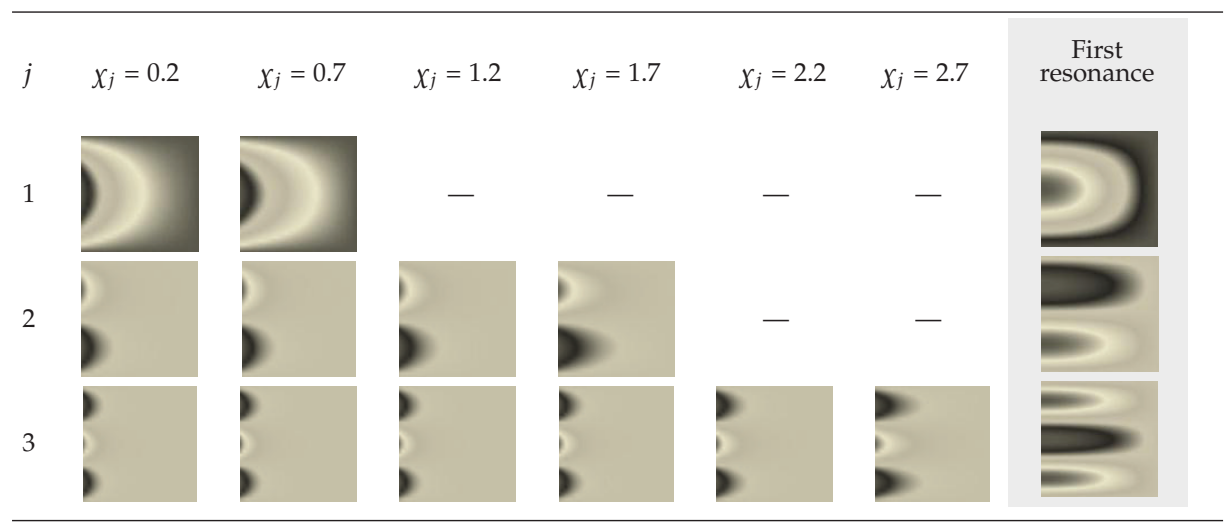

Table 11: Domains division for $j=1$ and $m=1$.

\begin{tabular}{cccc}
\hline Region I & Region II & Critical point & Region III-a \\
$x_{j}<j$ & $j<x_{j}<x_{\text {critical }}^{1}$ & $x_{\text {critical }}^{1}$ & $x_{\text {critical }}^{1}<x_{j}<x_{\text {zero }}^{1}$ \\
$x_{j}=0.2$ & $x_{j}=1.05$ & $x_{j}=\sqrt{5} / 2$ & $x_{j}=1.3$ \\
\hline & & & \\
\hline & Trigonometric & First resonance & Trigonometric \\
\hline
\end{tabular}

Table 12: Domains division for $j=1$ and $m=3$.

\begin{tabular}{cccc}
\hline Zero point & Region III-b & Critical point & Region III-a \\
$X_{\text {zero }}^{1}$ & $x_{\text {zero }}^{1}<x_{j}<x_{\text {critical }}^{2}$ & $x_{\text {critical }}^{2}$ & $x_{\text {critical }}^{2}<x_{j}<x_{\text {zero }}^{2}$ \\
$X_{j}=x_{\text {zero }}^{1}$ & $x_{j}=1.6$ & $x_{j}=\sqrt{13} / 2$ & $x_{j}=2.1$ \\
\hline & & & \\
\hline & & & \\
\hline
\end{tabular}

to the left (Region II). When $x_{j}$ tends to $x_{\text {critical }}^{1}$, the corresponding mode shape approaches the first resonance configuration. For $x_{\text {critical }}^{1}<x_{j}<x_{\text {zero }}^{1}$, the mode shape resembles the first resonance configuration dislocated to the right (Region III-a). When $x_{j}$ tends to $\chi_{\text {zero }}^{1}$ the corresponding mode shape approaches a null pressure condition at the interface, defining the first zero point. Table 11 illustrates these domains for $j=1$ and $m=1$, resulting in $x_{\text {critical }}^{1}=\sqrt{5} / 2$.

Table 12 illustrates the next sequence, with $j=1$ and $m=3$, resulting in $x_{\text {critical }}^{2}=$ $\sqrt{13} / 2$. It is important to notice that Region III is divided into two categories. The first, classified as "a", is contained in the interval between the first resonance and the first zero. The second, classified as " $b$ ", is limited by the first zero and the second resonance. From the first resonance and beyond only Region III is present. The zero point is defined as the value of 


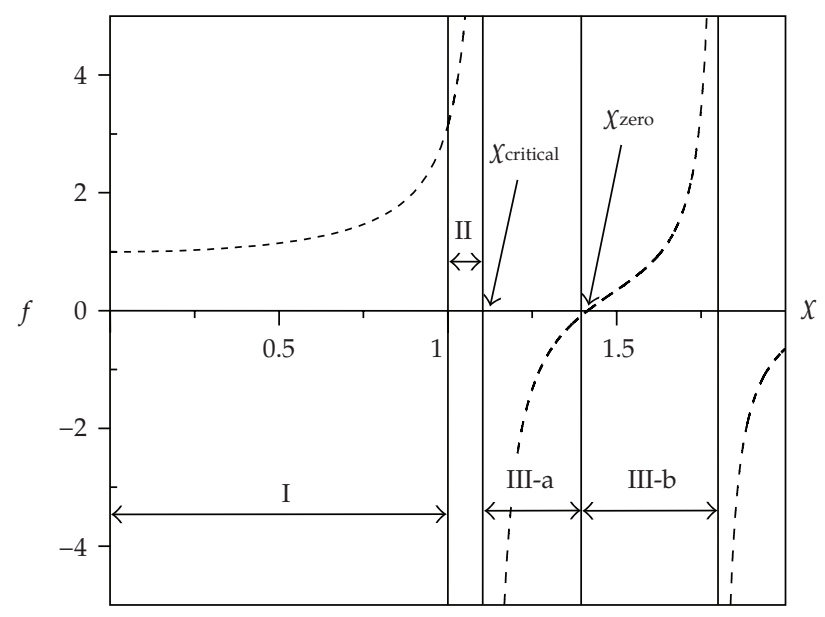

Figure 10: Variation of $f(x, j)$ along the $\chi$ axis for values of $j=1$ and $r=1$.

$x_{j}$ which establishes a null pressure condition at the interface $\left(\xi_{x}=0\right)$. Analysis of expressions (3.15) and (3.17) indicates that this situation occurs whenever $f_{1}=0$. Thus,

$$
f_{1}=\sin \left[-r \pi \sqrt{x^{2}-j^{2}}\right]=0
$$

Solution of the above expression results in

$$
\chi_{\text {zero }}=\sqrt{\left(\frac{\bar{m}}{r}\right)^{2}+j^{2}}, \quad \bar{m}=1,2,3 \ldots
$$

which indicates exactly the same expression given by (4.32) when $r=1$. Therefore, it is possible to conclude that the previous described common points in the parametric abacuses are equivalent to the zero points, defining conditions of null pressure at the interface and consequently coupled frequencies equal to the corresponding in-vacuum values.

It is also possible to divide the generalized fluid-added mass diagram of Figure 3 into the previously described regions. Figure 10 illustrates these results. Region II defines the beginning of the trigonometric solution and corresponds to the final interval before the first resonance. The first critical point establishes a signal change in the amplitude factor $f$ of (4.24), which becomes negative. This factor remains negative until the first zero point, defining Region III-a. From this point until the second resonance the amplitude factor is kept positive, defining Region III-b. After the second resonance the cycle restarts, alternating between Regions III-a and III-b. The negative values of $f$ have the physical meaning of an added stiffness, while the positive values define an additional mass. Therefore, solutions located on Regions I, II, and III-b provide the effects of an extra mass to the system, while solutions located in Region III-a provide an extra stiffness. Tables 11 and 12 indicate that solutions in Region III-a are given by critical configurations dislocated to the right, while solutions in Region III-b are equivalent to resonant modes dislocated to the left. Based on the previous analysis it is possible to conclude that solutions located at any of the previous 
described domains are equivalent to modified uncoupled cavity modes, with hyperbolic or trigonometric variations, and with corresponding configurations dislocated in the horizontal direction when compared to the uncoupled cavity solution.

\subsection{Results Discussion for Analysis 2}

This type of analysis presents an infinite summation in the generalized fluid-added mass term for even and odd values of $j$. Therefore, even values of this parameter have a common set of resonances (antisymmetrical cavity modes). This is also true for odd values of $j$ (symmetrical cavity modes). The occurrence of this phenomena implies in composed modes, where a given structural mode shape has more than a single $j$ related to $\phi(y)$, with the moving boundary at $S 1$ resulting in a combination of even or odd values of $j$. In some cases, especially in the first set of modes, a dominant mode is present and the proposed procedure is able to identify related frequencies (e.g. modes 1, 2, and 4,). However, the procedure fails if no significant dominance is present (as it can be noticed on mode 6). It is also important to notice that for an entirely open cavity (Analysis 1) there is no occurrence of common resonances, since summation is reduced to a non trivial solution only for $n=j$, resulting in a unique set of critical values for each $j$. Parametric abacuses could also be built for this type of analysis, resulting in similar conclusions to those identified in Analysis 1.

\section{Concluding Remarks}

A closed analytical procedure for solution of frequencies and mode shapes of a simple beam connected to an open rectangular acoustic cavity was presented. The mathematical development resulted in a frequency equation defined by two dimensionless parameters, which enabled the construction of practical abacuses for solution and interpretation of the coupled problem. Analytical results are in agreement with analysis using the finite element method and indicate the presence of two distinct categories of solutions. The first type presents hyperbolic nature and a strong influence of the structure, producing typical added mass modes, with coupled frequencies inferior to the corresponding structural in-vacuum values. The second type presents a higher influence of the cavity modes, with coupled frequencies which can be smaller, greater, or even equal to the corresponding structural in-vacuum values. Mode configurations from this last category are equivalent to modified uncoupled cavity modes, with strong similarity in both modes and frequencies depending on the values of $\mathcal{F}_{1}$ and $\mathcal{F}_{2}$,

The proposed solution is useful for interpretation of the coupled phenomena, providing an understanding of the modes sequences and corresponding configurations. The resulting mathematical expressions applied at the practical abacuses allow the identification of these general conclusions, with an initial interval $x<j$, resulting in added mass modes, followed by regions with $x>j$, where the coupled solution can assume conditions related to the structural in-vacuum frequencies values, such as, added stiffness (higher), added mass (smaller), or a null pressure at the interface (equal). The limit curve identified by $x^{2}$ enables the identification of these regions. Solutions located above this function present characteristics of added mass modes, while solutions located under this curve present an added stiffness behavior. For a given mode $j$ only a single hyperbolic solution is possible (typical added mass mode), followed by an infinite set of trigonometric solutions (modified cavity modes). 
As an extension, a simplified solution for a closed cavity in the transversal direction was also developed. The mathematical expressions indicate that summation is present in this type of solution, defining, respectively, symmetrical and antisymmetrical cavity resonances for odd and even values of $j$. Practical consequences of this phenomena imply composed mode shapes, with $\phi(y)$ represented by a set of odd or even values of $j$. The proposed procedure is able to identify coupled frequencies whenever a dominant mode is present. However, this technique fails when solutions are related to composed modes. Properties of an entirely open cavity, such as added mass, added stiffness, or null pressures at the interface are also valid in this case.

Although results have been presented for two specific cases, solutions involving other types of boundary conditions for both the structure and the cavity are also possible, resulting in similar expressions and abacuses for solution and interpretation of this phenomena. It is important to remember that the only limitation of this procedure is the prior knowledge of the imposed deformation functions at the interface.

\section{Acknowledgment}

The authors are grateful for the financial support provided by CNPq scholarships.

\section{References}

[1] K. J. Bathe, “Fluid-structure interactions," Mechanical Engineering Magazine, vol. 120, no. 5, pp. 66-68, 1998.

[2] R. B. Davis, Techniques to assess acoustic-structure interaction in liquid rocket engines, Ph.D. thesis, Department of Mechanical Engineering and Materials Science, Duke University, 2008.

[3] F. Fahy, Foundations of Engineering Acoustics, Academic Press, London, UK, 1st edition, 2001.

[4] E. H. Dowell and H. M. Voss, "The effect of a cavity on panel vibrations," AIAA Journal, vol. 1, pp. 476-477, 1963.

[5] A. J. Pretlove, "Free vibrations of a rectangular panel backed by a closed rectangular cavity by a closed rectangular cavity," Journal of Sound and Vibration, vol. 2, no. 3, pp. 197-209, 1965.

[6] R. W. Guy and M. C. Bhattacharya, "The transmission of sound through a cavity-backed finite plate," Journal of Sound and Vibration, vol. 27, no. 2, pp. 207-216, 1973.

[7] E. H. Dowell, G. F. Gorman III, and D. A. Smith, "Acoustoelasticity: general theory, acoustic natural modes and forced response to sinusoidal excitation, including comparisons with experiment," Journal of Sound and Vibration, vol. 52, no. 4, pp. 519-542, 1977.

[8] J. Pan and D. A. Bies, "The effect of fluid-structural coupling on sound waves in an enclosuretheoretical part," Journal of the Acoustical Society of America, vol. 87, no. 2, pp. 691-707, 1990.

[9] K. L. Hong and J. Kim, "Analysis of free vibration of structural-acoustic coupled systems-part I: development and verification of the procedure," Journal of Sound and Vibration, vol. 188, no. 4, pp. 561-575, 1995.

[10] H. Goto and K. Toki, "Vibrational characteristics and seismic design of submerged bridge piers," Memoirs of the Faculty of Engineering (Kyoto University), vol. 27, pp. 17-30, 1965.

[11] C.-Y. Liaw and A. K. Chopra, "Earthquake response of axisymmetric tower structures surrounded by water," UCB/EERC Report 73-25, Earthquake Engineering Research Center, University of California, Berkeley, Calif, USA, 1973.

[12] Y. Zhu, Z. Weng, and J. Wu, "The coupled vibration between column and water considering the effects of surface wave and compressibility of water," Acta Mechanics Sinica, vol. 10, pp. 657-667, 1989.

[13] J. T. Xing, W. G. Price, M. J. Pomfret, and L. H. Yam, "Natural vibration of a beam-water interaction system," Journal of Sound and Vibration, vol. 199, no. 3, pp. 491-512, 1997.

[14] H. Lamb, "On the vibrations of an elastic plate in contact with water," Proceedings of the Royal Society of London, vol. A98, pp. 205-216, 1921. 
[15] M. Amabili and M. K. Kwak, "Free vibrations of circular plates coupled with liquids: revising the lamb problem," Journal of Fluids and Structures, vol. 10, no. 7, pp. 743-761, 1996.

[16] Y. K. Cheung and D. Zhou, "Coupled vibratory characteristics of a rectangular container bottom plate," Journal of Fluids and Structures, vol. 14, no. 3, pp. 339-357, 2000.

[17] A. K. Chopra, "Earthquake response of concrete gravity dams," Tech. Rep. EERC 70-1, Earthquake Engineering Research Center, University of California, Berkeley, Calif, USA, January 1970.

[18] A. Rashed, Dynamic analyses of fluid-structure systems, Ph.D. thesis, California Institute of Technology, Pasadena, Calif, USA, 1983. 


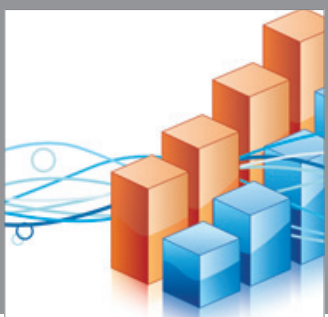

Advances in

Operations Research

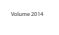

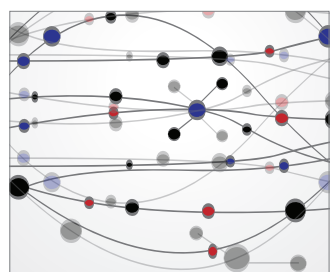

\section{The Scientific} World Journal
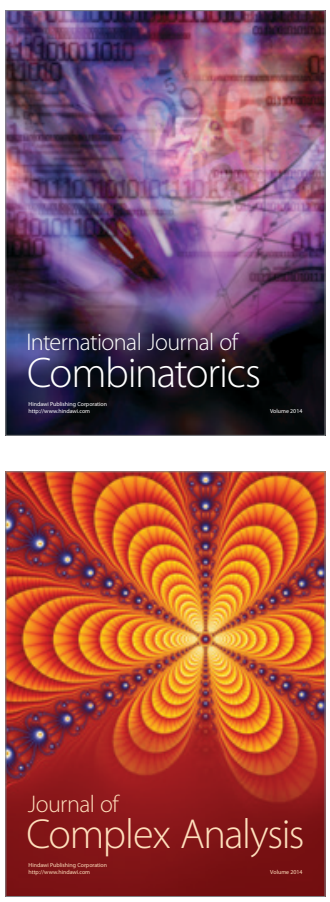

International Journal of

Mathematics and

Mathematical

Sciences
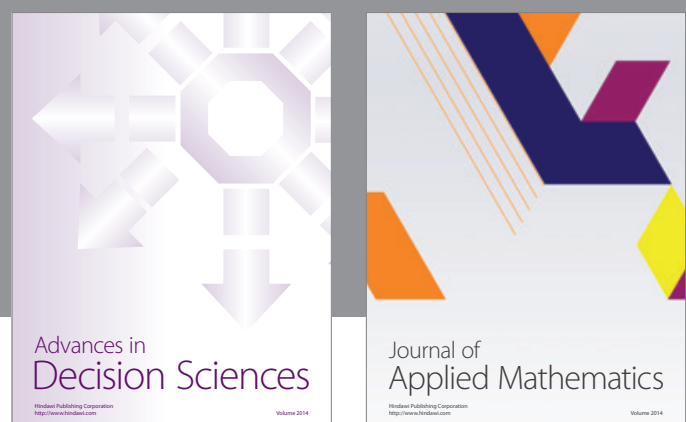

Journal of

Applied Mathematics
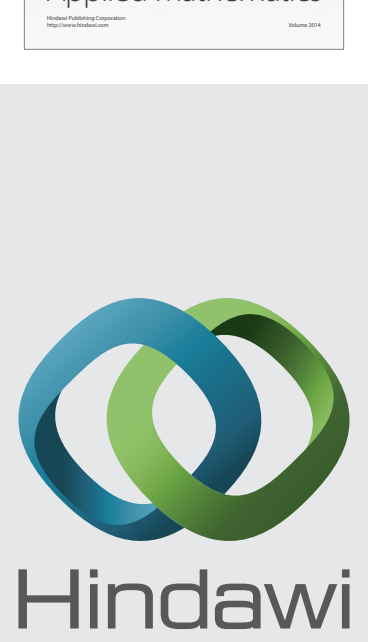

Submit your manuscripts at http://www.hindawi.com
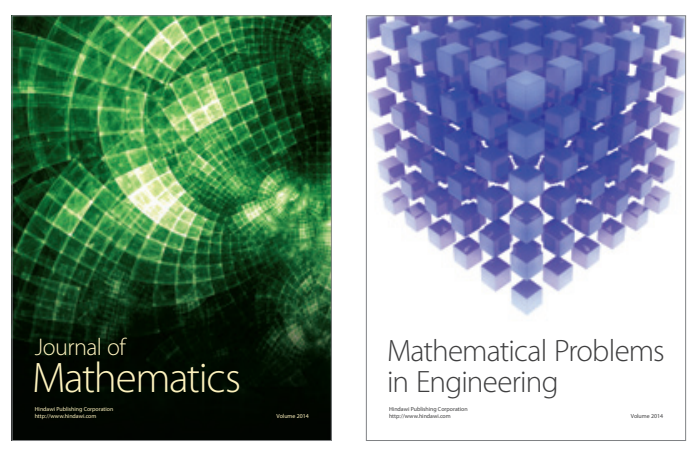

Mathematical Problems in Engineering
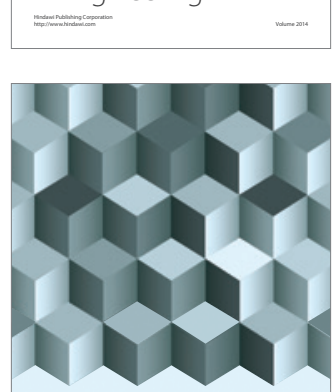

Journal of

Function Spaces
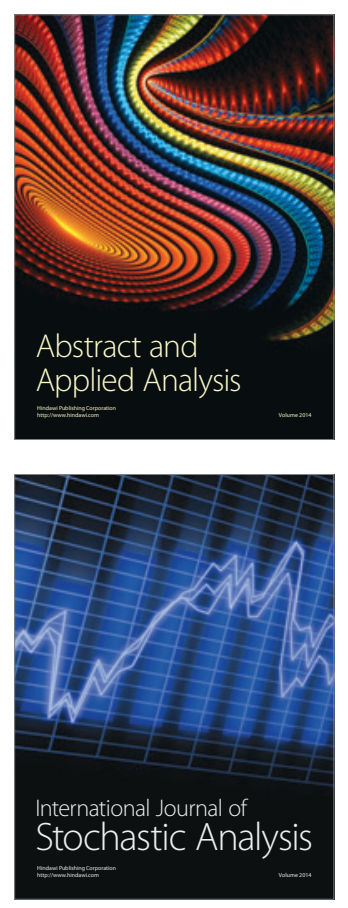

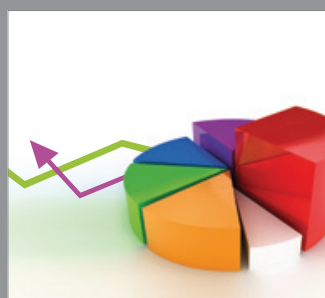

ournal of

Probability and Statistics

Promensencen
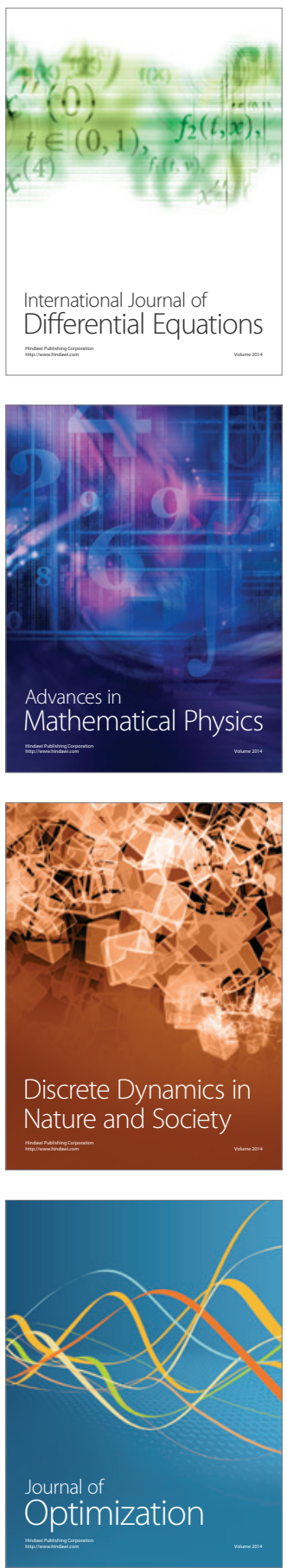